\title{
CRISI ECONOMICA, ISTITUZIONI \\ E RENDIMENTO IN 17 DEMOCRAZIE
}

\author{
di Adriano Pappalardo
}

Alla metà degli anni settanta, la prima di una serie di crisi economiche colpiva le democrazie occidentali, sottoponendo a sfide più o meno severe le loro strutture e le loro capacità politico-istituzionali. Del tema si è occupata un'intera letteratura, che ha fornito ragguardevoli contributi alla conoscenza del contenuto e dell'efficacia delle risposte di singoli paesi o gruppi di paesi e degli adattamenti/mutamenti subiti dalle società e dagli attori interessati ${ }^{1}$. In questo articolo, viene tracciato un bilancio della materia, aggiornato con informazioni e spiegazioni che si estendono fino alla metà degli anni novanta e coprono diciassette paesi.

In una parola, ciò che interessa è il rendimento della democrazia in condizioni di sfida alla stabilità e al funzionamento delle sue istituzioni, e questo comporta, naturalmente, una precisa specificazione di queste istituzioni, dei loro meccanismi decisionali e degli esiti associati. Per intendersi con un esempio, il nostro universo comprende quattro casi (Danimarca, Finlandia, Norvegia, Svezia) a lungo considerati «campioni di rendimento» (Rowthorn e Glyn 1990, 21) per i loro bassi tassi di disoccupazione, buoni livelli di crescita, elevata spesa pubblica e surplus di bilancio, nel contesto rimarchevolmente stabile di società omogenee, sistemi partitici di pluralismo moderato, governi dominati o egemonizzati dalla sinistra e forme di rappresentanza degli interessi altamente centralizzate e cooperative (o neocorporative). Comprensibilmente, dunque, queste caratteristi-

Questo articolo è una versione ridotta e leggermente modificata della mia introduzione al volume Democrazie e decisioni (Roma, NIS, 1998), scritto con Liborio Mattina. S'intende che la responsabilità del presente testo è soltanto mia.

1 Rassegne recenti includono Keech, Bates e Lange (1991); Lange e Meadwell (1991); Schmidt (1996).

RIVISTA ITALIANA DI SCIENZA POLITICA / a. XXVII, n. 3, dicembre 1997 
che sono state spesso associate, a uno, o più, aspetti del contesto socio-politico-istituzionale utilizzati per spiegare l'andamento dell'una o l'altra variabile economica. Con intenti analoghi, le prossime pagine affrontano fatti alquanto diversi, in particolare il declino degli antichi campioni di rendimento, passati negli anni novanta dietro molti altri paesi per crescita, deficit, disoccupazione e attraversati parallelamente da una più o meno severa crisi politica.

È così confermato, da un lato, che le due dimensioni possono essere correlate; ma, d'altro canto, nuovi sviluppi e nuove conclusioni non sono senza effetto sugli schemi interpretativi circolanti nella letteratura, sulle variabili esplicative da privilegiare e sul trattamento dei dati forniti a sostegno di precedenti generalizzazioni. Specie di fronte ai cambiamenti subiti dai paesi scandinavi, infatti, molte di quelle generalizzazioni appaiono perlomeno problematiche, e non semplicemente perché superate dagli eventi. Il superamento è stato talvolta favorito, se non reso inevitabile, dalla tendenza ad estrapolare a medio-lungo termine da osservazioni rivelatesi congiunturali, nell'ambizione di costruire «grandi» (e altrettanto fragili) teorie; e - seconda e più grave fallacia - dati e relazioni fra i dati sono stati talaltra costruiti così da corroborare un'impressione di continuità, o stabilità, anche laddove processi di cambiamento già in corso avrebbero potuto essere rilevati con scelte teoriche e tecniche $\mathrm{di}$ analisi diverse.

Seguendo la raccomandazione di prestare maggiore attenzione a tali processi nella ricerca comparata (Keman 1993), provvederò a selezionare le variabili più promettenti per mettere in luce le dinamiche politico-istituzionali rivelate dai paesi trattati in tempi di crisi. La selezione verrà condotta e giustificata attingendo e, ove necessario, modificando, la vasta letteratura teorica ed empirica sulle determinanti del rendimento. Componendo le determinanti prescelte in un indice additivo, sarà poi possibile ottenere una graduatoria di paesi parzialmente diversa da altre e - credo - più attuale e più compatibile con l'andamento delle variabili dipendenti. Queste ultime, dal canto loro, sono formate dalle serie di indicatori macroeconomici comunemente usati per misurare il rendimento, anche se la presente analisi è la più inclusiva finora tentata e la prima a presentare i dati sotto forma di trends, cioè di andamenti nel tempo. L'intenzione è, ancora una volta, di sottolineare processi di cambiamento e, specificamente, se e come è andata aumentando (o riducendosi) l'effica- 
cia delle risposte alla crisi, se efficacia o inefficacia sono concomitanti empiricamente con determinate configurazioni politicoistituzionali e se l'eventuale concomitanza è teoricamente congrua, cioè ragionatamente imputabile a tali configurazioni.

\section{Modelli di democrazia}

La politica conta? Non conta? Quanto? E in quali condizioni? Sinteticamente, i rapporti fra democrazie e rendimento vertono su interrogativi come questi, da tempo all'attenzione della letteratura. In un primo - e tuttora più cospicuo - filone di ricerca, oggetto degli interrogativi è stato definire se e che differenza faccia il partito al potere per il governo dell'economia e, specificamente, per l'andamento di disoccupazione, inflazione, crescita, spesa pubblica e, soprattutto, spesa sociale. Inaugurato dagli studi pionieristici di Hibbs (1977), Tufte (1978), Castles e McKinley (1979), Cameron (1984), questo filone è partito da ipotesi relativamente semplici per approdare a modelli complessi, nei quali la composizione politica del governo è stata via via associata a condizioni di prosperità o di crisi, alla distribuzione delle «risorse di potere» fra classi sociali, alla forza dell'opposizione, alle scadenze elettorali, alla vulnerabilità internazionale dell'economia e, in breve, a ogni sorta di fattore suscettibile di influenzare la politica economica e i suoi esiti. Un numero crescente di test econometrici proporzionatamente sofisticati ha così prodotto grande abbondanza di risultati, specificazioni, approfondimenti, di sicuro informativi, ma al tempo stesso poco discriminanti, poco coerenti, se non contraddittori, scarsamente cumulativi. Per esempio, diversi tipi di partiti (socialisti e democristiani) sono stati trovati associati allo stesso effetto (espansione della spesa sociale), in contrasto con la differenziazione attesa in base a semplicistiche dicotomie sinistra/destra; varie riformulazioni della dicotomia per tener conto di una complessità maggiore del previsto hanno dato luogo a una pluralità di indicatori scarsamente intercorrelati e, come tali, generatori di effetti differenziati (Schmidt 1996, 160-162), buoni per dimostrare tutto e il contrario di tutto; altre differenze, talvolta drastiche, sono poi emerse quando si sono variati i paesi, il periodo (preo post-shocks petroliferi) e l'arco di tempo considerati; e sofisticate polemiche tecniche sono infuriate sui modelli econometrici usati (Hicks e Patterson 1989; Garrett e Lange 1989; Jackman 
1989), ovviamente a sostegno di conclusioni empiriche opposte.

Nel complesso, il bilancio è che non vi sono correlazioni significative fra $\mathrm{i}$ partiti al potere e indicatori di rendimento come inflazione, disoccupazione e crescita (Schmidt 1996, 166); minor disoccupazione, semmai, è «predetta» più accuratamente dalla forza del sindacato e dal neocorporativismo (Hicks 1988; Crepaz 1992), ma di sicuro non negli anni novanta secondo i nostri dati; i partiti, a loro volta, tornano a far differenza per la spesa sociale se accompagnati da un vasto complesso di altre condizioni (Swank 1988; Hicks, Swank e Ambuhl, 1989; Hicks e Misra 1993; Huber, Ragin e Stephens 1993), ma non altrettanto quando si considera la spesa civile totale (Blais, Blake e Dion 1993); e tassazione, deficit e debito pubblico, infine, sono certo manipolati politicamente, ma non riflettono tanto i programmi, o il «colore» dei governi, quanto i vincoli o incentivi strutturali entro i quali essi operano (Roubini e Sachs 1989a e 1989b; Siniscalco e Tabellini 1993; Borrelli e Royed 1995).

Siano o no soddisfacenti per concludere che i partiti contano, questi dati provano senz'altro un'influenza parziale e lacunosa, che non migliora molto quando si cerca di potenziare la spiegazione con una qualche teoria delle «risorse di potere», o del «compromesso di classe», includente il contributo al rendimento di sindacati forti e centralizzati e/o sistemi concertati (neocorporativi) di policy-making (Korpi e Shalev 1979; Korpi 1983; Lehmbruch e Schmitter 1982; Streeck e Schmitter 1985). Con questo, dicevo, si fanno progressi sulla disoccupazione, ma si rimane ben al di qua di spiegazioni inclusive, sostanzialmente perché le classiche varianti di tali teorie accentuano indebitamente la capacità degli attori collettivi di far valere i propri obiettivi programmatici. Il loro presupposto «volontaristico» ha preso, quindi, ad essere messo in discussione da una letteratura recente, sempre più orientata a riconoscere che partiti e forze sociali sono «onnipresenti, ma non onnipotenti» perché, per trasmettere preferenze o scelte di chi governa in risultati conformi, le «risorse» degli, o la «concertazione» fra gli, attori non bastano senza il sostegno strutturale di norme costituzionali, regole elettorali, sistemi partitici, ecc., appropriati al raggiungimento di quei risultati. Poiché queste ed altre proprietà pertinenti variano internazionalmente, si è aperto così un nuovo filone di ricerca rivolto ad identificare le differenze ritenute più significative, a classificare di conseguenza i paesi interessati e a valutarne l'impatto sul rendimento. 
Di queste analisi, la tabella 1 fornisce una sinossi, non esauriente, ma rappresentativa. Come si vede, le variabili politicoistituzionali che si vuole «contino» sono a prima vista molte, anzi troppe, rispetto alle dimensioni del rendimento che sono rivolte a spiegare. Tecnicamente, tuttavia, la maggior parte degli autori provvede a ridurre la complessità fornendo indici compositi per semplice addizione dei punteggi attribuiti ad ogni variabile, $\mathrm{o}$, talvolta, tramite l'analisi fattoriale. Questi più parsimoniosi strumenti esplicativi sono fondamentalmente tributari di un'unica «teoria», o, più precisamente, sono costruiti a partire dai modelli di democrazia (maggioritario e consensuale) brillantemente sintetizzati e operazionalizzati da Lijphart più di dieci anni fa (1984). Questi modelli, che lo stesso Lijphart utilizzerà più tardi per l'analisi del rendimento (vedi tabella), forniscono soluzioni istituzionali opposte a una proprietà al riguardo importante - il livello di concentrazione del potere. Nella soluzione maggioritaria, o «modello Westminster», la concentrazione è massimizzata, e una pluralità di effetti attesi è condivisa dalla maggior parte degli autori elencati nella tabella e altri ancora: isolamento dell'esecutivo da pressioni parlamentari ed elettorali, di forze sociali organizzate o «punti di veto» istituzionali; conseguente maggior spazio di manovra per decisioni «chiare e ferme» ed, eventualmente, per una politica della «mano forte»; tempestività d'azione, capacità di innovare, imporre sacrifici, fissare priorità, allocare risorse selettivamente, coordinare obiettivi confliggenti e porre in essere le scelte fatte (Colomer 1995; Huber, Ragin e Stephens 1993; Immergut 1992; Lijphart 1994a; Schmidt 1996; Weaver e Rockman 1993).

Con varie accentuazioni e distinguo, insomma, istituzioni maggioritarie sono indicate come il veicolo appropriato di politiche conformi alle intenzioni degli attori responsabili; e questo grazie alla minimizzazione dei «costi decisionali» (Buchanan e Tullock 1962) garantita da un ristretto numero di decidenti, o, appunto, dalla concentrazione del potere nelle mani della maggioranza di governo. Viene così evitato il tipico problema del modello alternativo (quello consensuale), nel quale la dispersione del potere impone invece decisioni molto più inclusive $\mathrm{e}$, al limite, all'unanimità, associate a contrattazioni lunghe e lente, rigidità e garanzie procedurali, compromessi poco innovativi, o, nel peggiore dei casi, la perpetuazione dello status quo per effetto del veto dell'una o l'altra minoranza coinvolta. D'altro canto, a queste ben note inefficienze, l'inclusività unisce un vantaggio 
TAB. 1. Il rendimento delle istituzioni. Cause e conseguenze

\begin{tabular}{|c|c|c|c|c|c|c|c|}
\hline \multirow[t]{2}{*}{ Autori } & \multirow[t]{2}{*}{ Variabili indipendenti } & \multirow[t]{2}{*}{ Variabili dipendenti } & \multicolumn{2}{|c|}{ Relazione } & \multirow[t]{2}{*}{ Paesi } & \multirow[t]{2}{*}{ Periodi } & \multirow[t]{2}{*}{ Trattamento dei dati } \\
\hline & & & attesa & verificata & & & \\
\hline $\begin{array}{l}\text { Roubini e Sachs } \\
\text { (1989a; 1989b) }\end{array}$ & 1. Numero di partiti al governo & $\begin{array}{l}\text { 1. Spesa/PNL } \\
\text { 2. Deficit/PNL }\end{array}$ & $\begin{array}{l}+ \\
+\end{array}$ & $\begin{array}{l}+ \\
+\end{array}$ & $\begin{array}{l}13 \\
15\end{array}$ & $\begin{array}{l}1961-1985 \\
1972-1985\end{array}$ & Quantitativo \\
\hline $\begin{array}{l}\text { Huber, Ragin e Stephens } \\
\text { (1993) }\end{array}$ & $\begin{array}{l}\text { 1. Federalismo } \\
\text { 2. Presidenzialismo } \\
\text { 3. Bicameralismo } \\
\text { 4. Sistema maggioritario } \\
\text { 5. Referendum }\end{array}$ & 1. Spesa sociale/PIL & $\begin{array}{l}- \\
- \\
- \\
-\end{array}$ & $\begin{array}{l}- \\
- \\
- \\
-\end{array}$ & 17 & $1956-1988$ & 3 Quantitativo \\
\hline Siniscalco e Tabellini (1993) & $\begin{array}{l}\text { 1. \% voti ai partiti di governo } \\
\text { 2. Numero di crisi di governo } \\
\text { 3. Rapporto voti/seggi } \\
\text { 4. Indice di frammentazione } \\
\text { del governo }\end{array}$ & $\begin{array}{l}\text { 1. Debito/PIL } \\
\text { 2. Inflazione/crescita } \\
\text { 3. Indice di miseria } \\
\text { 4. Indice di inefficienza } \\
\text { dei servizi }\end{array}$ & $\begin{array}{l}+ \\
+ \\
+ \\
+\end{array}$ & $\begin{array}{l}+ \\
+ \\
+ \\
+\end{array}$ & 21 & $1970-1991$ & Quantitativo \\
\hline $\begin{array}{l}\text { Weaver e Rockman } \\
\text { (1993b) }\end{array}$ & $\begin{array}{l}\text { 1. Separazione dei poteri } \\
\text { 2. Coalizioni } \\
\text { 3. Sistemi a partito } \\
\text { predominante } \\
\text { 4. Bicameralismo } \\
\text { 5. Federalismo } \\
\text { 6. \% voti ai partiti di governo }\end{array}$ & $\begin{array}{l}\text { 1. Deficit/PIL } \\
\text { 2. Politiche pensionistiche } \\
\text { 3. Politiche industriali }\end{array}$ & & & $\begin{array}{l}\text { Case studie } \\
\text { comparati }\end{array}$ & sVari anni & Qualitativo \\
\hline Lijphart (1994a) & $\begin{array}{l}\text { 1. Sistemi proporzionali } \\
\text { 2. Democrazie consensuali }\end{array}$ & $\begin{array}{l}\text { 1. Disoccupazione } \\
\text { 2. Inflazione } \\
\text { 3. Crescita }\end{array}$ & $\begin{array}{l}- \\
\overline{+}\end{array}$ & $\begin{array}{l}\overline{-} \\
\overline{? 2}\end{array}$ & $\begin{array}{l}13 \\
18\end{array}$ & $1961-1988$ & 3 Quantitativo \\
\hline
\end{tabular}


TAB. 1. (segue)

\begin{tabular}{|c|c|c|c|c|c|c|c|}
\hline \multirow[t]{2}{*}{ Autori } & \multirow[t]{2}{*}{ Variabili indipendenti } & \multirow[t]{2}{*}{ Variabili dipendenti } & \multicolumn{2}{|c|}{ Relazione } & \multirow[t]{2}{*}{ Paesi } & \multirow[t]{2}{*}{ Periodi } & \multirow[t]{2}{*}{ Trattamento dei dati } \\
\hline & & & attesa & verificata & & & \\
\hline Colomer (1995) & $\begin{array}{l}\text { 1. Numero effettivo di partiti } \\
\text { 2. Bicameralismo } \\
\text { 3. Presidenzialismo } \\
\text { 4. Decentramento }\end{array}$ & 3 & 3 & & 17 & 3 & 3 \\
\hline Crepaz (1996) & $\begin{array}{l}\text { 1. \% voti ai partiti } \\
\text { di governo }\end{array}$ & $\begin{array}{l}\text { 1. Disoccupazione } \\
\text { 2. Inflazione } \\
\text { 3. Indice di miseria }\end{array}$ & $\begin{array}{l}- \\
- \\
-\end{array}$ & $\begin{array}{l}- \\
- \\
-\end{array}$ & 18 & $1961 / 1988$ & Quantitativo \\
\hline Schmidt (1996) & $\begin{array}{l}\text { 1. Indice dei vincoli } \\
\text { al potere centrale }\end{array}$ & $\begin{array}{l}\text { 1. Spesa totale/PIL } \\
\text { 2. Spesa corrente/PIL }\end{array}$ & - & - & 19 & $1960 / 1994$ & Quantitativo \\
\hline
\end{tabular}

1 Relazioni miste, non discriminanti, variabili con le politiche interessate e con un'ampia gamma di circostanze contingenti.

2 Relazioni confermate parzialmente e debolmente.

3 Non specificato.

4 Include le seguenti variabili: Bicameralismo, Federalismo, Costituzione rigida, Refciendum, Partecipazione all'UE, Autonomia della Banca centrale. 
altrettanto noto, ossia la minimizzazione dei «costi esterni» (Sartori 1974; Lehner 1988, 88-90) che possono ricadere sugli esclusi da decisioni a maggioranza e possono provocarne reazioni tali da mettere a dura prova la legittimità e la continuità dell'azione di governo. Al riguardo, dunque, Weaver e Rockman (1993a, 458) fanno presente che «il punto debole di un'elevata capacità di innovazione è il rischio di drastici rovesciamenti di politica quando il controllo passa di mano fra i partiti», com'è nella logica maggioritaria dell'alternanza. E Lijphart, a sua volta, sottoscriveva nel 1977 (47-52) la «credenza convenzionale» che il power sharing consensuale assicurasse maggior rappresentatività a spese dell'effettività e dell'efficienza; ma oggi (1994a, 12) anch'egli ritiene una «condotta continua» preferibile alla «mano forte», e decisioni unanimi più «sagge», e con maggiori probabilità di successo, di decisioni rapide prese contro la «volontà di importanti settori della società».

L'elenco potrebbe proseguire con gli argomenti analoghi proposti da Crepaz (1996) in base ad un'interpretazione piuttosto sui generis di Olson. Ma poiché il banco di prova dei pro e contro dei due modelli di democrazia è ovviamente empirico, il passo preliminare opportuno è una verifica delle opposte posizioni, che verranno poi riprese nel bilancio conclusivo. Per questa verifica, si utilizzeranno gran parte delle variabili indipendenti presentate nella tabella 1 , con qualche esclusione e qualche aggiunta che possono essere adeguatamente giustificate. Nella sostanza - si è ricordato - tutti gli autori attingono al contributo di Lijphart, che rimane la trattazione di gran lunga più soddisfacente degli indicatori di democrazia maggioritaria o consensuale, e della logica sottintesa di concentrazione/frammentazione del potere. Del suo contributo, pertanto, l'analisi incorpora le variabili più importanti, che comprendono il sistema elettorale, o, meglio, i suoi effetti sulle due successive (sistema partitico e tipo di governo), i rapporti di forza esecutivo-legislativo, il grado di centralizzazione del sistema politico, la struttura del parlamento e, nella versione più aggiornata, le forme (pluralistica o neocorporativa) di rappresentanza degli interessi (Lijphart 1984, 4-9 e 1994a; Lijphart e Crepaz 1991).

In breve, si tratta di sette dimensioni analitiche, più di quante ne usino gli autori in tabella e lo stesso Lijphart. Dalla sua recente ricerca sul rendimento, infatti, rimangono fuori la centralizzazione (cioè, l'alternativa Stato unitario/federale) e la struttura del parlamento (unicamerale o bicamerale); ma l'esclu- 
sione si deve a motivi tecnici ed è debole sul piano teorico-concettuale, perché federalismo e bicameralismo (specie se «forte») sono plausibilissimi veicoli di frammentazione del potere, come tali del tutto pertinenti al criterio chiave sul quale viene valutata l'efficienza nel dibattito appena riassunto. Si può, perciò, concordare con i molti che nella tabella 1 inseriscono l'uno o l'altro, o entrambi, fra i loro explanantes, mentre Lijphart torna a sua volta nel giusto quando ignora altre proprietà utilizzate da alcuni di questi autori, Schmidt in particolare. Mi riferisco alle costituzioni «rigide», alla pratica del referendum, e all'autonomia della banca centrale, che egli considera elementi «contromaggioritari», o «vincoli al potere dei governi nazionali» (1996, 172-173), peraltro senza spiegare perché. E, viceversa, una spiegazione è necessaria e, se cercata, non conferma l'influenza delle tre variabili. $\grave{E}$ vero, infatti, che costituzioni rigide richiedono per una serie di atti maggioranze qualificate e complesse procedure decisionali, ossia il più ampio consenso. Ma che ciò vincoli le politiche di spesa pubblica (come sostiene Schmidt) è semplicemente infondato, perché queste politiche sono governate in tutte le democrazie industriali dalla legislazione ordinaria, per la quale basta la maggioranza semplice. E il referendum, a sua volta, è normalmente proibito in materia finanziaria e di bilancio (con l'eccezione solitaria della Svizzera), e non è nemmeno in principio «contromaggioritario», ma, al contrario, «pro-egemonico» (Lijphart 1984, 201-204; Papadopoulos 1995) nei paesi interessati (tutti, meno Italia e Svizzera) che ne riservano l'iniziativa ai partiti al potere. Come dire che la validità teorica dei due indicatori è zero, mentre quella dell'autonomia della banca centrale appare quantomeno dubbia ${ }^{2}$. E poiché, inoltre, i loro presunti effetti variano assai poco nell'universo, è scontato che l'indice dei vincoli al potere centrale di Schmidt deluda anche empiricamente, cioè spieghi poco o nulla degli andamenti della spesa pubblica.

Meglio, invece, si può fare tornando alle sette dimensioni già ricordate. Di queste, tuttavia, contributi diversi forniscono le versioni operazionali ritenute di volta in volta più convenienti e, per conto mio, farò lo stesso, modificando, quando necessa-

2 L'effetto dell'autonomia della banca centrale è dubbio per la sua ambiguità. Come sostenuto da Streeck (1994) nel caso tedesco, infatti, l'autonomia impone ai governi un «vincolo» (al rigore di bilancio) quasi sempre benvenuto e utilizzato come uno strumento in più per perseguire $i$ propri obiettivi. 
rio, questo o quell'indicatore, $\mathrm{o}$, eventualmente, aggiungendone di nuovi. Per quanto possibile, comunque, mi sforzerò di evidenziare non solo le differenze politico-istituzionali fra sistemi maggioritari e consensuali, ma anche i processi di cambiamento subiti da ciascun paese nel corso del tempo. Questa, infatti, è un'esigenza tanto trascurata dalla letteratura, quanto inderogabile in un periodo marcato da un evidente ed elevato dinamismo, che è occultato dall'uso corrente di classificare in base ai valori medi delle variabili rilevanti in tutto il, o parte del, dopoguerra. A quest'uso, avallato anche da Lijphart ${ }^{3}$, sostituirò perciò presentazioni dei dati rivolte per quanto possibile a cogliere tendenze e in base ad esse posizionerò vari paesi sul continuum maggioritario-consensuale alquanto diversamente dai suoi (e altrui) contributi, nonostante il ricorso a molti degli stessi indicatori.

\section{Dai modelli ai dati}

L'universo da esaminare comprende diciassette democrazie consolidate, da distinguere fra sistemi maggioritari e sistemi consensuali. Due differenze cruciali fra questi sistemi concernono il numero e la distribuzione dei seggi fra i partiti, che sono a loro volta in gran parte effetto di altrettante variabili del sistema elettorale: formula e soglia «effettiva» di rappresentanza (Lijphart 1994b). La prima differenza dipende soprattutto dalla soglia - quanto più alta, tanto meno partiti - mentre una determinata formula (maggioritario secco) è associata pressoché perfettamente alla produzione di maggioranze assolute, due terzi delle quali «fabbricate artificialmente» a partire da minoranze elettorali. In breve, cioè, da queste regole dipendono il formato (bi- o multipartitico) e il tipo di governo (monopartitico o di coalizione) del sistema, ovvero le proprietà chiave per qualsiasi analisi della concentrazione o framinentazione del potere e delle conseguenze associate. A rigore, fierciò, non è errato tentare di cogliere concentrazione o frammentazione (o, in altre parole, lo spartiacque maggioritario/consensuale) per via di una semplice dicotomia fra plurality system e regimi proporzionali, come fatto inizialmente da Lijphart (1994a). Ma la strategia non è la mi-

3 Nel saggio del 1994, infatti, Lijphart continua ad utilizzare i dati della ricerca di dieci anni prima, a loro volta medie 1945-1980. 
TAB. 2. Sistemi partitici

\begin{tabular}{|c|c|c|c|c|c|c|c|}
\hline \multirow[b]{2}{*}{ Paesi } & \multicolumn{2}{|c|}{ Formato } & \multicolumn{5}{|c|}{ Stabilità } \\
\hline & $\begin{array}{l}\text { N. effettivo } \\
\text { di partiti }\end{array}$ & $\begin{array}{l}\text { N. partiti } \\
\text { principali }\end{array}$ & $\begin{array}{c}\text { Trend } \\
1965-1996\end{array}$ & $\begin{array}{c}\text { Media } \\
1945-1965\end{array}$ & $\begin{array}{c}\text { Media } \\
1965-1996\end{array}$ & $\begin{array}{c}\text { Media } \\
1975-1996\end{array}$ & $\begin{array}{c}\text { Media } \\
1985-1996\end{array}$ \\
\hline Australia & $2,6-2,4$ & $4-4$ & $-0,125$ & 5 & 6,4 & 6,5 & 5,8 \\
\hline Austria & $2,1-3,5$ & 3.5 & 0,227 & 5,2 & 5,6 & 6 & 8,9 \\
\hline Belgio & 5.8 & $6-11$ & 0,05 & 9,4 & 9,2 & 9,4 & 10 \\
\hline Canada & $2,7-2,4$ & $4-5$ & 0,783 & 10 & 12,3 & 13,9 & 22,7 \\
\hline Danimarca & $4,1-4,6$ & $5-8$ & $-0,022$ & 8,7 & 11,4 & 11,8 & 8,9 \\
\hline Finlandia & $5-4,9$ & $7-8$ & 0,108 & 5 & 9,3 & 9,4 & 11,2 \\
\hline Francia & $2,5-2,9$ & 5.5 & 0,315 & 16,3 & 10,9 & 11,9 & 13,6 \\
\hline Germania & $2,7-2,9$ & $4-5$ & 0,067 & 12,4 & 6,6 & 6,6 & 7,8 \\
\hline Giappone $^{1}$ & $2,5-2,9$ & $5-6$ & 0,11 & 9,6 & 5,8 & 5,5 & 7,1 \\
\hline Gran Bretagna & $2-2,3$ & 3.4 & $-0,064$ & 4,6 & 6,1 & 6,4 & 3,8 \\
\hline Italia & $3,5-6,4$ & $7-9$ & 0,468 & 12,7 & 11,7 & 13,6 & 18,2 \\
\hline Norvegia & $3,2-4$ & $6-7$ & 0,198 & 4,8 & 11,1 & 12,2 & 11,7 \\
\hline Nuova Zelanda 2 & $2-2,2$ & 3.3 & 0,308 & 4,9 & 9,9 & 11,5 & 13,8 \\
\hline Olanda & $5,8-5,1$ & $10-7$ & 0,171 & 5,2 & 10,7 & 11,2 & 12 \\
\hline Stati Uniti & $2-2$ & $2-2$ & $-0,012$ & 4 & 3,4 & 3,2 & 3 \\
\hline Svezia & $2,8-3,5$ & 5.7 & 0,254 & 5 & 7,5 & 8,3 & 10,2 \\
\hline Svizzera & $5,1-5,6$ & $8-7$ & 0,132 & 3,3 & 6,3 & 6,9 & 7,8 \\
\hline
\end{tabular}

1 Trend e medie fino al 1993

2 Formato, trend e medie fino al 1993

Nota: Il calcolo del numero effettivo di partiti (Laakso e Taagepera 1979) è relativo all'ultima elezione degli anni sessanta e all'ultima elezione degli anni novanta. Sono stati considerati come partiti principali quelli con almeno il $2 \%$ dei voti validi (stessi anni del precedente indicatore).

Fonti: Bardi (1996); Bartolini e Mair (1990); EJPR (vari anni), calcoli propri.

gliore possibile perché rende difficile classificare casi importanti, mette indebitamente nello stesso mazzo varianti diverse di proporzionale e distingue, sì, bipartitismo e governo monopartitico da multipartitismo e coalizioni, ma non fra le altrettanto eterogenee versioni della seconda categoria. Poiché tutte queste distinzioni sono invece di grande rilevanza per il rendimento, informazioni più ricche e più precise debbono e possono essere acquisite esaminando sistemi partitici e tipi di governo per conto proprio, a cominciare dai dati della tabella 2 .

La prima osservazione che si impone è la conferma dell'impulso al formato bipartitico dato dal maggioritario secco, o da varianti strettamente affini ${ }^{4}$. Tutti e sei i paesi interessati ( $\mathrm{Au}$ -

4 Le varianti affini sono il voto alternativo (Australia) e il maggioritario a doppio turno (Francia), ai quali Lijphart (1994b) attribuisce la stessa soglia «effettiva» di rappresentanza del maggioritario secco $(35 \%)$. 
stralia, Canada, Francia, Gran Bretagna, Nuova Zelanda, Stati Uniti) mostrano infatti un numero «effettivo» di partiti inferiore a tre; poiché il numero effettivo è ponderato per i seggi, esso è un indicatore di concentrazione del potere più accurato dei «partiti principali» (col. 2) - che include quelli con almeno il $2 \%$ dei voti; ma anche se si contano i voti, i valori restano bassi e, soprattutto, pressoché invariati in un periodo caratterizzato da un elevato dinamismo nella maggioranza degli altri paesi.

Il panorama, viceversa, è molto più variegato quando si passa ad esaminare l'universo proporzionale: entro di questo, solo Germania e Giappone mantengono a lungo termine valori paragonabili a quelli maggioritari; all'opposto, tre paesi (Finlandia, Olanda e Svizzera) mostrano livelli stabili, ma molto elevati, di frammentazione della rappresentanza, mentre un quarto (Italia) accentua marcatamente il suo storico pluralismo estremo; ${ }^{5}$ e una frammentazione diversificata, ma comunque crescente, accomuna infine Austria, Belgio, Danimarca, Norvegia e Svezia, portando anch'essi ai livelli record del dopoguerra.

Ovviamente, a questa varietà di andamenti corrispondono tipi diversi di proporzionale, che, tuttavia, non bastano per spiegarne direzione ed intensità. E non bastano perché, a differenza del maggioritario, «la proporzionale è un sistema elettorale debole» e, se «davvero 'puro', ...senza effetto: lascia ai partiti la libertà di moltiplicarsi al negativo, semplicemente perché non ne penalizza la moltiplicazione. Ma la libertà di moltiplicarsi non è causa (causa efficiente) di moltiplicazione più di quanto la libertà di viaggiare sia causa del viaggiare» (Sartori 1984, 24), e quel che sposta, piuttosto, è un'altra variabile - la strutturazione del sistema partitico. Strutturazione, infatti, significa che la scena politica è dominata da partiti di massa dotati di saldi legami ideologici e organizzativi con l'elettorato e, come tali, in grado di controllarlo capillarmente e di sottrarlo alla conquista di vecchi e nuovi competitori. Quale che sia la debolezza manipolativa della proporzionale, quindi, la frammentazione non ne è un esito scontato, in presenza dell' «effetto bloccante, o effetto controbilanciante, di un sistema partitico forte». Se c'è, esso diventa «il fattore causale» di un formato ridotto e stabile (Sartori 1984, 28), mentre instabilità, frammentazione, accesso e succes-

5 Come è noto, la riforma semimaggioritaria italiana del 1993 è stata ampiamente «proporzionalizzata», ciò che spiega il persistere della frammentazione. Cfr. Bartolini e D'Alimonte (1995). 
so di competitori andranno di pari passo con la crisi, comunque prodottasi, dei partiti di massa. E questo significa che la prima metà della tabella sottintende, e si completa con, i dati della seconda metà, i quali utilizzano appunto una misura atta a cogliere l'efficienza del controllo organizzativo dell'elettorato e «l'effetto bloccante» associato.

La misura è il classico indicatore degli spostamenti di voti fra i partiti (instabilità, o volatilità, totale netta) ed ha come tale un ovvio e ben documentato rapporto con il loro livello di strutturazione (Bartolini e Mair 1990). Quanto minori gli spostamenti, infatti, tanto più i partiti si possono supporre «forti», cioè capaci di canalizzare gli impulsi della società ed, eventualmente, di isolarsi da pressioni elevate al mutamento e alla frammentazione. Anche se non è il solo indicatore di strutturazione, né dipende solo da questa (Bartolini e Mair 1990; Katz e Mair 1994), una bassa volatilità è associata, quindi, sia alla concentrazione della rappresentanza che all'autonomia dall'elettorato degli attori democratici chiave, cioè a due caratteristiche di intrinseco interesse per l'analisi del rendimento nel contesto dei modelli maggioritario e consensuale. Dato questo importante ruolo, i dati sono forniti nella tabella in dettaglio e calcolati in due diversi modi: sotto forma di trends temporali (all'aumento, alla diminuzione, alla stagnazione dell'instabilità); e per valori medi, rivolti a cogliere quel che la prima misura può travisare: ad esempio, l'ovvia differenza fra due tendenze molto marginalmente ascendenti (Belgio e Germania), ma da livelli ben altrimenti alti e bassi. Trends e medie, poi, sono calcolati all'incirca nel periodo che ci interessa e le seconde estese, inoltre, all'intero dopoguerra. È possibile così un'ispezione sistematica entro e fra paesi, nonché delle variazioni intervenienti fra tempi di crisi e tempi di prosperità. $\mathrm{Ma}$, comunque si leggano i dati, gli andamenti che emergono nell'universo proporzionale sono a ricalco del numero dei partiti. Infatti, dietro al formato ridotto di Germania e Giappone c'è una debole tendenza all'aumento dell'instabilità entro i limiti dei livelli medi di gran lunga più bassi, sia rispetto agli altri paesi che alla prima parte del dopoguerra; nonostante le recenti difficoltà del partito predominante giapponese e l'enorme potenziale di frammentazione introdotto dalla riunificazione tedesca, la «forza canalizzante» dei due sistemi non appare dunque seriamente in discussione. Tutt'altra situazione, invece, nei nove paesi restanti, i cui partiti sono alle prese con elettorati sempre più volatili, che ne denunciano decre- 
scenti capacità di controllo e crescente destrutturazione; questi processi sono talvolta segnalati dalle medie ma non dai trends (Belgio, Danimarca), semplicemente perché i paesi interessati mantengono livelli relativamente invariati di elevata instabilità per gran parte del dopoguerra; talaltra, invece, trends più o meno accentuati sono accompagnati da medie 1965-1996 doppie, o più che doppie, rispetto al 1945-1965 (Finlandia, Norvegia, Olanda, Svizzera); un terzo gruppo, a sua volta, si distingue dal precedente solo per l'accentuazione del trend e il ritmo più veloce di crescita dell'instabilità media nell'ultimo decennio (Austria, Svezia); e l'una e l'altro, infine, raggiungono le punte massime in Italia, dove i partiti storici si sono letteralmente dissolti per effetto di una crisi di transizione tuttora in corso.

Tutti e nove i paesi, insomma, seguono un proprio percorso (più o meno marcato, lento o accelerato, continuo o discontinuo), ma in una direzione comune: sistemi partitici sempre più deboli, più frammentati, più a rimorchio della società che agenti «canalizzatori». Sommandosi alla proporzionale, questa debolezza ha prodotto i cambiamenti di formato indicati nella prima parte della tabella, cancellando consolidati sistemi di pluralismo limitato, o ridimensionando drasticamente gli storici partiti predominanti di Norvegia e Svezia. A maggior ragione, la tenuta giapponese e tedesca spicca come riprova dell' «effetto controbilanciante» della strutturazione. $\mathrm{Ma}$, naturalmente, gli esempi canonici di stabilità provengono dai sistemi elettorali maggioritari, che sono per definizione «forti» (cioè, capaci di mantenere un formato bipartitico) e tali si confermano nella tabella: pienamente, laddove trends addirittura discendenti e medie di instabilità via via più basse rivelano una sinergia di effetto elettorale ed effetto ristrutturazione (Australia, Gran Bretagna, Stati Uniti); più relativamente in Canada, Francia e Nuova Zelanda, poiché le due variabili lavorano ora l'una contro l'altra: il maggioritario continuando a mantenere ridotto il formato, mentre i partiti fanno fatica a controllare un elettorato molto mobile, vanno incontro, talvolta, a crisi davvero disastrose (Canada), o sono costretti a riforme radicali per prevenirle (Nuova Zelanda) ${ }^{6}$.

Questi esempi documentano che, per quanto forte il sistema elettorale, l'isolamento e l'autonomia delle élites dalla società non sono garantite, se esse ricevono pressioni da destrutturazio-

6 Mi riferisco alla débâcle del partito conservatore alle elezioni del 1993 (Canada) e alla rivoluzionaria introduzione della proporzionale in Nuova Zelanda. 
ne inusualmente elevate e persistenti. Ma ciò non toglie la differente resistenza delle istituzioni rispetto alle democrazie consensuali; e non toglie che la differenza si estenda all'altra, ricordata, dimensione dei modelli di Lijphart - il tipo di governo. Come mostra la tabella 3, infatti, solo ai quattro plurality systems (Canada, Gran Bretagna, Nuova Zelanda, Stati Uniti) sono associate, quasi senza eccezioni, maggioranze monopartitiche (o governi monocratici, nel caso del presidente americano), tutte «fabbricate» artificialmente da minoranze elettorali. Da questa concentrazione massima del potere, inoltre, si discosta poco l'Australia, se si tiene conto che le sue frequenti coalizioni si fondano sull'alleanza elettorale permanente dei partiti liberale e conservatore. E le stesse coalizioni sopramisura francesi, a loro volta, sono meno frammentate di quanto sembrino, in parte perché anche il maggioritario a doppio turno obbliga i loro membri a candidature comuni nei collegi, e in parte per la spinta coesiva proveniente (salvo cobabitation) dalla presidenza elettiva della repubblica (Lijphart 1994b, 143).

Fra i sistemi proporzionali, d'altro canto, solo Giappone e Germania sono nettamente sovrarappresentate nelle prime due categorie della tabella, cioè fra i governi monopartitici o fra le minime coalizioni vincenti. Questi paesi confermano così una vocazione «maggioritaria» sui generis, già implicita nel formato ridotto del sistema partitico e ora riflessa nei due tipi di esecutivo che Lijphart $(1984,10-11)$ associa al «modello Westminster». Con qualche distacco, dovuto a una maggior proporzione di tipi più frammentati, seguono Austria e Olanda, che sono nondimeno interessanti per la comune tendenza a coalizioni minime più frequenti dal 1965, o 1975, rispetto all'insieme del dopoguerra (Lijphart 1984, 61; Woldendorp, Keman e Budge 1993, 113). Ma questa tendenza non si manifesta in Italia (storicamente ancorata ai tipi 3-4-5), né in Svizzera, e si rovescia nelle rimanenti democrazie: in Belgio e Finlandia, con lo svuotamento post-1965 delle categorie 1 e 2 (già ben popolate) a favore delle coalizioni sopramisura; in Norvegia e Svezia, con un analogo spostamento, più accentuato dal 1975, verso le minoranze monopartitiche e le coalizioni minoritarie; in Danimarca, con la netta progressione di queste ultime sul tipo 4 , che rimane comunque fortemente rappresentato.

In gran parte dell'universo proporzionale, quindi, l'aumento post-1965 del numero dei partiti sembra aver comportato più frammentazione dei governi, oppure minoranze monocolore 
TAB. 3. Tipi di governo: $1965-1996 ; 1975-1996$

\begin{tabular}{|c|c|c|c|c|c|c|c|c|c|c|c|c|}
\hline \multirow[t]{2}{*}{ Paesi } & \multicolumn{2}{|c|}{$\begin{array}{l}\text { Maggioranza } \\
\text { monopartitica }\end{array}$} & \multicolumn{2}{|c|}{$\begin{array}{l}\text { Coalizione minima } \\
\text { vincente }\end{array}$} & \multicolumn{2}{|c|}{$\begin{array}{l}\text { Coalizione } \\
\text { sovramisura }\end{array}$} & \multicolumn{2}{|c|}{$\begin{array}{c}\text { Minoranza } \\
\text { monopartitica }\end{array}$} & \multicolumn{2}{|c|}{$\begin{array}{c}\text { Coalizione } \\
\text { di minoranza }\end{array}$} & \multicolumn{2}{|c|}{$\begin{array}{c}\text { Governo } \\
\text { di transizione }\end{array}$} \\
\hline & $1965-96$ & $1975-96$ & $1965-96$ & $1975-96$ & $1965-96$ & $1975-96$ & $1965-96$ & $1975-96$ & $1965-96$ & 1975.96 & $1965-96$ & $1975-96$ \\
\hline Australia & 3 & 3 & 6 & 1 & & & & & & & & \\
\hline Austria & 2 & 1 & 2 & 2 & 2 & 1 & 1 & & & & & \\
\hline Belgio & & 6 & 3 & 9 & 6 & & 2 & 1 & 2 & 2 & & \\
\hline Canada & 7 & 6 & & & & & 4 & 1 & & & & \\
\hline Danimarca & & & 2 & 1 & & & 6 & 3 & 5 & 5 & & \\
\hline Finlandia & & & 3 & 1 & 13 & 9 & 1 & & 2 & 2 & 1 & \\
\hline Francia & 1 & 1 & 3 & 1 & 10 & 5 & 3 & 3 & 1 & & 1 & 1 \\
\hline Germania & & & 4 & 2 & 1 & & & & & & & \\
\hline Giappone & 10 & 8 & 1 & 1 & 3 & 2 & 2 & 2 & 1 & 1 & & \\
\hline Gran Bretagna & 1a 6 & 4 & & & & & 1 & & & & & \\
\hline Italia & & & 5 & 3 & 15 & 9 & 5 & 2 & 2 & 2 & 2 & 2 \\
\hline Norvegia & & & 2 & 1 & & & 8 & 6 & 3 & 2 & & \\
\hline Nuova Zelanda & 8 & 5 & & & & & & & & & & \\
\hline Olanda & & & 6 & 4 & 4 & 2 & & & & & 3 & 1 \\
\hline Stati Uniti & 7 & 5 & & & & & & & & & & \\
\hline Svezia & 2 & & 2 & 2 & & & 6 & 5 & 2 & 2 & & \\
\hline Svizzera & & & & & 9 & 6 & & & & & & \\
\hline
\end{tabular}

Nota: Nei governi sono conteggiati solo i partiti titolari di ministeri. Un governo si considera cambiato solo se cambiano tali partiti e/o il primo ministro; l'intervento di elezioni, pertanto, è considerato irrilevante se non accompagnato da tali cambiamenti e se non modifica il tipo di governo. Inoltre, non sono conteggiati i governi che, entrati in crisi, siano rimasti in carica con parte dei membri in attesa dell'insediamento del successivo esecutivo, né le compagini governative composte esclusivamente da tecnici. A queste regole si è derogato per la Svizzera, i cui governi non sono sfiduciabili dal parlamento e sono presieduti a turno (per un anno) da uno dei quattro partiti che dal 1959 formano la coalizione al potere. Per evitare di inflazionare artificiosamente $\mathrm{i}$ dati, in questo caso si è quindi calcolata la durata sulla scadenza elettorale, che, con le variazioni di seggi che comporta, approssima il valore politico degli indicatori usati per gli altri paesi. I dati degli Stati Uniti si riferiscono, invece, alle amministrazioni presidenziali, riportate nella prima colonna in quanto considerate equivalenti delle maggioranze monopartitiche. Il periodo coperto va dal $1^{\circ}$ gennaio 1965 (1975) a tutto il 1996, esclusi i governi eventualmente formati nel corso di quest'ultimo anno.

Fonti: Katz e Mair (1992); Morlino (1998); Woldendorp, Keman e Budge (1993), calcoli propri. 
TAB. 4. Durata media dei governi (giorni)

\begin{tabular}{lrrr}
\hline Paesi & $1965-1996$ & $1975-1996$ & $1985-1996$ \\
\hline Australia & 1.265 & 2.472 & 2.039 \\
Austria & 1.621 & 1.924 & 1.348 \\
Belgio & 638 & 670 & 1.013 \\
Canada & 1.031 & 1.148 & 1.461 \\
Danimarca & 899 & 999 & 876 \\
Finlandia & 583 & 642 & 876 \\
Francia & 615 & 730 & 730 \\
Germania & 2.337 & 4.017 & 4.382 \\
Giappone & 683 & 569 & 479 \\
Gran Bretagna & 1.670 & 2.009 & 2.191 \\
Italia & 378 & 407 & 407 \\
Norvegia & 950 & 893 & 1.013 \\
Nuova Zelanda & 1.451 & 1.902 & 1.434 \\
Olanda & 964 & 1.148 & 1.461 \\
Stati Uniti & 1.670 & 1.607 & 1.461 \\
Svezia & 951 & 862 & 957 \\
Svizzera & 1.260 & 1.282 & 1.190 \\
\hline
\end{tabular}

Nota: La data di inizio è quella di formazione di un nuovo governo, se occorsa nell'anno indicato in testa alle colonne, o, in mancanza, il $1^{\circ}$ gennaio. La data finale è quella di sostituzione del governo, se occorsa durante il 1996, oppure il 31 dicembre. In mancanza di informazioni sull'ultimo governo postelettorale, si è utilizzata la data delle elezioni. Per la Svizzera, si è tenuto conto dell'elezione prossima di inizio e fine periodo. Anni e mesi di 365 e 30 giorni.

Fonti: EJPR (vari anni); Keesings Publications (vari anni); Woldendorp, Keman e Budge (1993), calcoli propri.

sempre più lontane dalla maggioranza e, come tali, implicitamente «frammentate» perché costrette a costruirla e ricostruirla continuamente con il sostegno occasionale di mutevoli coalizioni parlamentari. Rispetto a precedenti classificazioni, questo segnala una divaricazione crescente del modello consensuale dal modello Westminster, benvenuta metodologicamente perché una maggiore variabilità può avere più nitide ricadute sul rendimento, che diversi autori ritengono, appunto, decrescente al crescere dei negoziatori delle politiche pubbliche, entro il governo, o, se esso è minoritario, direttamente in parlamento (Roubini e Sachs 1989a e 1989b; Schick 1993; Edin e Ohlsson 1991). Questa posizione, però, non è condivisa da altri (Strom 1990; Crepaz 1996), i quali rifiutano di identificare tout court frammentazione e debolezza decisionale e sottolineano che di tale debolezza può essere maggiormente responsabile una variabile complementare - la stabilità (in termini di durata) degli 
esecutivi. Per Lijphart $(1984,80)$, anzi, proprio la durata rappresenta l'indicatore appropriato dei rapporti di forza fra questi e il parlamento, che è - si ricorderà - un'altra delle sue dimensioni dell'alternativa maggioritario/consensuale. Per tenerne conto, quindi, ho riportato nella tabella 4 i dati pertinenti che, ancora una volta, rivelano più variabilità dei calcoli di Woldendorp, Keman e Budge $(1993,108)$, specie nei sottoperiodi 1975-1996 e 1985-1996. Questa variabilità è in gran parte effetto di una diversa operazionalizzazione, poiché la tabella non vincola la durata alle scadenze elettorali, se esse non danno luogo a cambiamenti dei partiti al potere e/o del primo ministro ${ }^{7}$. Così facendo, viene appropriatamente colta la prolungata egemonia conservatrice (o laburista) in Germania, Gran Bretagna, Stati Uniti e Australia, cioè l'ininterrotta durata transelettorale dei governi Kohl, Thatcher/Major, Reagan, Clinton e Hawke, ed è posta una linea di demarcazione netta e strutturalmente rilevante rispetto a governi che transelettorali sono sempre meno (come in Norvegia e Svezia, a seguito della crisi del partito predominante), o non lo sono mai stati.

Pur senza eliminare alcune incongruenze, questo permette di far risaltare il solitario record tedesco, con la sua interminabile coalizione cristiano-liberale indiscutibilmente «predominante» sul parlamento, secondo la concettualizzazione di Lijphart (1984, 78ss.). Degna di nota, fra i sistemi proporzionali, è pure la durata dei governi austriaci (seppur fortemente declinante rispetto all'insieme del dopoguerra) e, in misura minore e ristretta al 1985-1996, di quelli olandesi ${ }^{8}$. Ma, di regola, e a rinforzo delle tendenze pre-1965, la parte alta della classifica è occupata da cinque sistemi maggioritari (Australia, Canada, Gran Bretagna, Nuova Zelanda, Stati Uniti), con una sola eccezione (Francia), peraltro sicuramente esagerata dall'inadeguatezza dell'indicatore per un sistema semipresidenziale (Lijphart 1984, 82-83). Tutti gli altri paesi ricadono sotto una soglia minima $(1.000$ giorni), seppure con notevoli variazioni, talvolta difficili da in-

7 In altre parole, il criterio adottato è diverso da quello di Woldendorp et al. (1993), che delle scadenze elettorali tengono conto, ma anche da quello di Lijphart (1984, 78 sgg.), che prende in considerazione il cambiamento di composizione politica del governo prescindendo da sostituzioni del primo ministro e appare, come tale, troppo restrittivo.

8 Sui generis, invece, la Svizzera, che non mostra variazioni fra periodi perché i governi non sono sfiduciabili e, dunque, si è dovuta usare la scadenza quadriennale fissa delle elezioni per determinarne la durata. 
terpretare: al Giappone, per esempio, la stima della forza dei governi in funzione della loro durata si addice almeno altrettanto poco che alla Francia ${ }^{9}$, anche se il fatto che essa si sia quasi dimezzata nel 1985-1996 rispetto alla media 1949-64 è un sintomo delle recenti difficoltà affrontate dal partito predominante; Belgio, Danimarca, Finlandia e Italia, a loro volta, sono stati modelli storici di debolezza e instabilità coalizionale, e tali rimangono con largo margine; ma particolarmente significativo è che, dappresso, seguano Svezia e Norvegia, perché questi paesi vantavano una tradizione di lunga durata; e dal 1965, invece, nel secondo c'è stato un declino di un buon $25 \%$ rispetto al precedente ventennio; mentre $\mathrm{i}$ governi svedesi hanno fatto peggio, attestandosi sul $35 \%$ medio in meno ${ }^{10}$.

La più accentuata divaricazione post-1965, dunque, proviene dalla combinazione dell'eccezionale exploit tedesco con la conferma, o i progressi, della stabilità dei governi maggioritari e con gli andamenti scadenti, o in rapido peggioramento, della maggior parte degli altri paesi. Conformemente - sosterrebbe Lijphart - questi ultimi hanno avuto esecutivi sempre più deboli, o meno forti, dominati dal, piuttosto che dominanti sul, parlamento e, in quanto tali, dotati di decrescente autonomia decisionale. Ma, per quanto condivisibile, il giudizio merita un'integrazione, perché la durata è un indicatore importante, ma non certo definitivo, e non solo nei casi difficili della Francia o del Giappone. Concettualmente, infatti, durare può essere condizione necessaria per operare con successo, ma non è detto serva granché senza un'adeguata coesione dei responsabili delle politiche pubbliche. Per guadagnare precisione, e per correggere qualche misclassificazione, dunque, occorre tener conto anche di questa variabile, le cui operazionalizzazioni, peraltro, sono difficili, controverse, spesso discutibilmente giustificate, quasi mai soddisfacenti per l'uso comparato, e specialmente per la comparazione nel tempo ${ }^{11}$. Senza pretendere di risolverle una volta per tutte, la tabella 5 presenta i risultati di una semplice misura, proposta da Siniscalco e Tabellini $(1993,815)$, che evi-

9 Salvo eccezioni, infatti, la rapida rotazione del primo ministro giapponese non sottintende significative crisi politiche, ma piuttosto la pratica di alternare le correnti del partito predominante alla guida del governo.

10 Calcoli propri sui dati di Woldendorp et al. (1993).

11 Questi problemi sono connessi all'uso di misurare la coesione in termini di polarizzazione, o di eterogeneità ideologico-programmatica dei partiti. Per la più importante letteratura in proposito, vedi Schmidt (1996). 
TAB. 5. Coesione del governo e coesione dell'opposizione

\begin{tabular}{|c|c|c|c|c|c|c|}
\hline \multirow[t]{2}{*}{ Paesi } & \multicolumn{2}{|c|}{$1965-1996$} & \multicolumn{2}{|c|}{$1975-1996$} & \multicolumn{2}{|c|}{$1985-1996$} \\
\hline & governo & opposizione & governo & opposizione & governo & opposizione \\
\hline Australia & 39,7 & 30,5 & 48,1 & 25,9 & 54,9 & 22,6 \\
\hline Austria & 42,9 & 25,3 & 36 & 30,8 & 32,3 & 27,4 \\
\hline Belgio & 17,6 & 8,3 & 14,2 & 6,8 & 13,8 & 7,2 \\
\hline Canada & 53,5 & 16,2 & 55,7 & 18,4 & 61,3 & 16 \\
\hline Danimarca & 25,4 & 16,4 & 19,6 & 11,4 & 14 & 11,8 \\
\hline Finlandia & 16,1 & 8,6 & 19,6 & 11,4 & 14 & 11,8 \\
\hline Francia & 30 & 14,7 & 33,9 & 14,9 & 42,5 & 11,7 \\
\hline Germania & 31 & 33,7 & 27,6 & 35,1 & 27,6 & 18,7 \\
\hline Giappone & 46,3 & 10,5 & 44,6 & 10,4 & 40,1 & 10,8 \\
\hline Gran Bretagna & 52,3 & 23,9 & 53 & 23,5 & 57,4 & 21,3 \\
\hline Italia & 21,3 & 9,6 & 18,8 & 9,9 & 16 & 7,4 \\
\hline Norvegia & 31 & 14,7 & 33,2 & 13,2 & 26,2 & 15,4 \\
\hline Nuova Zelanda & 57,6 & 42,4 & 57,8 & 42,2 & 56,5 & 43,5 \\
\hline Olanda & 20,6 & 5,7 & 23,6 & 5,8 & 27,2 & 6,8 \\
\hline Stati Uniti & 48,9 & 50,9 & 46,4 & 53,5 & 44,6 & 55,4 \\
\hline Svezia & 34,3 & 18,1 & 28,3 & 20,1 & 37,3 & 18,1 \\
\hline Svizzera & 20,4 & 3,4 & 20,1 & 3,4 & 19,7 & 3,5 \\
\hline
\end{tabular}

Nota: Non sono stati conteggiati i governi formati durante il 1996 e le altre categorie escluse nelle precedenti tabelle. Nel conteggio dei partiti, non si è tenuto conto degli «Altri». Quando il sostegno parlamentare di uno stesso governo (e la forza dell'opposizione) cambiano a seguito di elezioni, per il calcolo finale è stata utilizzata la media. Il dato degli Stati Uniti indica i rapporti di forza fra il partito del presidente in carica e l'opposizione.

Fonte: EJPR (vari anni); ES (vari anni); Gorvin (1989), Katz e Mair (1992), Woldendorp, Keman e Budge (1993), calcoli propri.

ta, o minimizza, quelle difficoltà, limitandosi a dividere la percentuale di seggi parlamentari del governo e dell'opposizione per il numero dei partiti che ne fanno parte ${ }^{12}$. Chiaramente, l'ipotesi sottintesa è che, ceteris paribus, i governi monopartitici maggioritari sono al vertice della gerarchia della coesione, specie se fronteggiati da opposizioni inefficaci perché frammentate. E possibile così controllare se la classificazione per durata, e anche per tipo di governo, sia rafforzata, ovvero relativizzata, dall'aggiunta di un terzo, più sensibile, indicatore. E il controllo rivaluta, per cominciare, le risorse dei governi francesi e giappo-

12 Mentre Siniscalco e Tabellini calcolano solo la coesione del governo, l'aggiunta della coesione dell'opposizione in questa sede viene effettuata in considerazione di un'ipotesi di Castles (1982) che condiziona la realizzabilità delle politiche sociali della sinistra alla forza del maggior partito di destra. Nella forma ora proposta, tale ipotesi è generalizzata al rendimento delle politiche pubbliche, che si suppone tanto maggiore quanto meno coesiva l'opposizione (di destra o di sinistra che sia), e viceversa. 
nesi, rispettivamente abbastanza e molto coesivi, e con un'opposizione invariabilmente divisa; ridimensiona, per contro, la posizione tedesca, per effetto di governi di coalizione ed opposizione unita, anche se non più nell'ultimo decennio; evidenzia il paradosso americano della divisione dei poteri fra presidente e Congresso di diverso colore; distingue la polarizzazione neozelandese fra maggioranze e minoranze ugualmente coesive dalla sempre più accentuata combinazione di monopartitismo maggioritario e minoranze mediamente, o molto, divise di Australia, Canada e Gran Bretagna; conferma l'estrema e crescente disarticolazione generale dei paesi già manifestamente più policentrici e instabili (Belgio, Danimarca, Finlandia, Italia); e rivela una varietà di tendenze intermedie nei rimanenti, sparpagliati fra il trio norvegese, olandese e svizzero di moderata coesione governativa e bassa coesione dell'opposizione e il maggior equilibrio fra le due in Austria e Svezia.

Come confermano i punteggi della tabella 8 , queste variazioni sono indipendenti quanto basta da tipo e durata dei governi per concludere che esse integrano il bilancio delle loro risorse con un genuino progresso informativo. L'informazione, però, è limitata a risorse strutturali strettamente politiche, mentre i governi operano, ovviamente, nel contesto di istituzioni e regole formalizzate disegnate, spesso intenzionalmente, per massimizzare, o minimizzare, la concentrazione del potere. Per questo, Lijphart ed altri distinguono i modelli maggioritario e consensuale anche in base al tipo di parlamento e alla forma di Stato (tab. 2), associando la massima concentrazione alla combinazione di monocameralismo e Stato unitario e la massima frammentazione al suo opposto (bicameralismo/federalismo). Conformemente, la tabella 6 riporta gli indicatori pertinenti, le cui definizioni operazionali sono selezionate dalla letteratura e danno luogo ad una graduatoria analoga di power sharing. A queste variabili usuali, però, ne abbiamo aggiunto due generalmente trascurate, ovvero utilizzate in modo insoddisfacente. La prima (in col. 1) verifica se la scelta del capo del governo sia di competenza parlamentare e, ovviamente, la esclude nella presidenza americana, della quale diamo in questa sede la definizione di Lijphart $(1984,85)$ : un'istituzione tipicamente maggioritaria, il cui potere concentrato e indipendente si contrappone al bicameralismo «forte» del Congresso ${ }^{13}$. A seguire, troviamo i si-

13 Al contrario, per Colomer (1995) e Huber, Ragin e Stephens (1993), la variabi- 
TАв. 6. Vincoli al potere dell'esecutivo 1975-1996

\begin{tabular}{|c|c|c|c|c|c|}
\hline \multirow[t]{2}{*}{ Paesi } & \multirow{2}{*}{$\begin{array}{l}\text { Scelta parlamentare } \\
\text { del premier }\end{array}$} & \multirow{2}{*}{$\begin{array}{l}\text { Potere } \\
\text { di sfiducia }\end{array}$} & \multicolumn{2}{|c|}{ Bicameralismo } & \multirow{2}{*}{$\begin{array}{c}\text { Decentramento } \\
\text { territoriale }\end{array}$} \\
\hline & & & forte & debole & \\
\hline Australia & & $\mathrm{XX}$ & $\mathrm{X}$ & & $\mathrm{X}$ \\
\hline Austria & $X$ & $X X$ & & & $\mathrm{X}$ \\
\hline Belgio 1 & $\mathrm{X}$ & $\mathrm{XX}$ & & $\mathrm{X}$ & \\
\hline Canada & & $\mathrm{XX}$ & & $\mathrm{X}$ & $\mathrm{X}$ \\
\hline Danimarca & $\mathrm{X}$ & $\mathrm{XX}$ & & & \\
\hline Finlandia & $\mathrm{X}$ & $\mathrm{XX}$ & & & \\
\hline Francia & & $\mathrm{X}$ & & & \\
\hline Germania & & $\mathrm{X}$ & $\mathrm{X}$ & & $\mathrm{X}$ \\
\hline Giappone & & $\mathrm{XX}$ & & $\mathrm{X}$ & \\
\hline Gran Bretagna & & $\mathrm{XX}$ & & & \\
\hline Italia & $\mathrm{X}$ & $\mathrm{XX}$ & $\mathrm{X}$ & & \\
\hline Norvegia & $\mathrm{X}$ & $\mathrm{XX}$ & & & \\
\hline Nuova Zelanda & & $\mathrm{XX}$ & & & \\
\hline Olanda & $\mathrm{X}$ & $\mathrm{XX}$ & & $\mathrm{X}$ & \\
\hline Stati Uniti & & & $\mathrm{X}$ & & $\mathrm{X}$ \\
\hline Svezia & $\mathrm{X}$ & $\mathrm{X}$ & & & \\
\hline Svizzera & $\mathrm{X}$ & & $X$ & & $X$ \\
\hline
\end{tabular}

1 Stato federale dal 1995.

Nota: La scelta del capo del governo è sottratta al parlamento (spazio in bianco) in caso di elezione diretta (Stati Uniti), o nomina da parte del presidente elettivo leader de facto della maggioranza (Francia), ovvero quando la legittimità a governare emerge automaticamente da elezioni-referendum sui candidati dei partiti o degli schieramenti in campo (sistemi a partito predominante, sistemi bipartitici, «democrazia del cancelliere» tedesca). Il potere parlamentare di sfiducia è assente negli Stati Uniti e in Svizzera (spazi bianchi), fortemente vincolato $(\mathrm{X})$ se il governo può sciogliere il parlamento e vigono la «sfiducia costruttiva» o altre restrizioni costituzionali (Francia, Germania, Svezia), moderatamente vincolato o incondizionato (XX) in tutti gli altri casi. Bicameralismo forte: poteri uguali o moderatamente disuguali delle camere; bicameralismo debole; poteri più accentuatamente asimmetrici; bicameralismo insignificante (in bianco, come il monocameralismo): poteri estremamente asimmetrici. Decentramento territoriale: federalismo o accentuato regionalismo.

Fonti: Colomer (1995); Gallagher, Laver e Mair (1995); Huber, Ragin e Stephens (1993); Schüttemeyer (1994).

stemi bipartitici o a partito predominante, il semipresidenzialismo francese e la «democrazia del cancelliere» tedesca, tutti accomunati da «elezioni referendum» sulla maggioranza e il suo capo, che li legittimano a governare finché un nuovo voto non li rimuova. Questo, infatti, apparenta le loro risorse al modello americano, o, perlomeno, le distingue nettamente da quelle di-

le presidente elettivo è un indicatore in più di pluralismo istituzionale e frammentazione decisionale. Chiaramente, dunque, in questa sede non si condivide la loro posizione. 
sponibili nei paesi che concentrano le condizioni «pure» del potere assembleare: coalizioni multipartitiche più o meno ampie, o monocolore ultraminoritari, scelti dopo il voto ed, eventualmente, a prescindere dal, o in contrasto col, suo risultato, e guidati da leader «fabbricati» in parlamento e totalmente dipendenti dal suo sostegno.

Fra $\mathrm{i}$ paesi in questione, peraltro, alcuni rientrano tipicamente nella definizione per tutto, o quasi tutto, il dopoguerra: Danimarca e Italia, in particolare. Ma, una volta di più, altri sono andati approdando alla categoria a partire dagli anni settanta, man mano che il declino del partito predominante minava la legittimità elettorale del primo ministro (Norvegia, Svezia), o diminuiva la coesione degli alleati sotto la sua guida (Austria, Belgio, Olanda), oppure la protezione del capo dello stato (Finlandia). Nel periodo che ci interessa, di conseguenza, la maggioranza tocca a questo gruppo, composto di governi comparativamente «deboli». E, certo, la debolezza è controbilanciata dal potere di scioglimento dell'assemblea vigente quasi dappertutto, salvo Finlandia e Norvegia e lo speciale caso svizzero (né potere di sfiducia, né potere di scioglimento); ma - come mostra la seconda colonna della tabella - di tale potere v'è comunque una graduazione significativa: nei sistemi multipartitici (meno la Svezia), esso è l'unico deterrente alla sfiducia, che si può definire perciò solo moderatamente vincolata; nel modello presidenziale, all'opposto, il vincolo è - salvo impeachment - ovviamente massimo; e, infine, è sicuramente rafforzato dalla «sfiducia costruttiva» o altre restrizioni costituzionali in Francia e Germania e cioè in due dei paesi con primo ministro legittimato elettoralmente. Anche se in parte relativizzato dai dati della terza e quarta colonna della tabella (per effetto del bicameralismo e del federalismo), il risultato è così un punteggio complessivo (tab. 8, col. 6) diverso da quello di altri autori e, secondo noi, più equilibrato e realistico: per esempio, perché assegna ai governi italiani e svizzeri più - e non meno - vincoli consensuali di quelli tedeschi; oppure, perché stima la Gran Bretagna più maggioritaria di Finlandia, Belgio o Danimarca, anziché viceversa; e, infine, perché rettifica un'ancora più bizzarra graduatoria Francia-Olanda, attribuendo alla seconda istituzioni più parlamentarizzate e policentriche della prima ${ }^{14}$.

14 Per l'una o l'altra di queste criticabili classificazioni, vedi Colomer (1995); Huber, Ragin e Stephens (1993); Schmidt (1996). 
Come che sia, vincoli al potere dell'esecutivo non provengono solo da regole formalizzate, ma anche da pratiche decisionali informali, quali quelle che si accompagnano a uno specifico sistema di rappresentanza e intermediazione degli interessi - il neocorporativismo (Lehmbruch e Schmitter 1982; Streeck e Schmitter 1985). Queste pratiche - il cui nocciolo è la concertazione delle politiche pubbliche con la partecipazione delle organizzazioni dei sindacati e del padronato - sono come tali «concettualmente affini», ed empiricamente correlate, alla «concertazione fra partiti» sottintesa dal modello consensuale (Lijphart e Crepaz 1991, 236). Di questo modello, in altre parole, il neocorporativismo rappresenta un'estensione, o un completamento, così come la formula speculare (il pluralismo) è associato alla competizione (anziché concertazione) fra stato e gruppi organizzati, cioè al tipico presupposto del modello maggioritario e dei suoi stili decisionali (Schmitter 1974; Lijphart 1984). Prevedibilmente, dunque, le stesse premesse e le stesse conclusioni sui meriti o demeriti di tale modello e della sua alternativa sono state fatte valere per i relativi sistemi di rappresentanza degli interessi, sui quali, anzi, il dibattito è stato più intenso e controverso che non sulle loro dimensioni politico-istituzionali. $\mathrm{Da}$ un lato, infatti, la concertazione neocorporativa è stata definita lo strumento ideale per governare società complesse, specialmente in tempi di crisi che richiedono il massimo impegno collettivo per la continuata produzione di un volume adeguato di beni pubblici. Tipicamente, questo successo è considerato effetto di un processo decisionale altamente centralizzato e inclusivo, in grado come tale di promuovere l'«interesse generale» e di minimizzare defezioni dal suo perseguimento (Berger 1981; Schmitter 1981; Goldthorpe 1984; Crouch 1985; Jankowski 1988). L'argomentazione a sostegno, sottintesa o esplicita, risale a Olson $(1965 ; 1982)$, per cui l'interesse generale è naturalmente «interiorizzato» dagli attori collettivi quando questi sono di dimensioni sufficientemente grandi da non poter scaricare su terzi i costi di eventuali comportamenti «irresponsabili». Poiché essi stessi (cioè, i loro seguaci) ne rimarrebbero vittime, segue un incentivo all'«efficienza da partecipazione», che taluni si spingono a considerare l'unica via all'ottimizzazione del rendimento economico (Magagna 1988; Scharpf 1991; Crepaz 1992). Ma a questa posizione Olson stesso obietterebbe che, con le dimensioni e il numero dei partecipanti, aumentano la complessità e la lentezza inerenti a risposte ampiamente consensuali, o, per riusare 
la terminologia di Buchanan e Tullock (1962), si incorre in crescenti «costi decisionali». Contrariamente alle precedenti previsioni, dunque, secondo altri proprio la sua inclusività espone la concertazione neocorporativa ad «inflessibilità e immobilismo» (Lehner 1988, 88-90), o, come conclude drasticamente Dahrendorf $(1989,139)$, la paluda dapprima di «un'apparenza di stabilità» destinata «a degenerare rapidamente in stagnazione... e dispute paralizzanti».

A rigore, fra opinioni così divergenti potrebbe scegliere la ricerca empirica, che sul tema è stata, però, abbondante quanto ambigua. Mentre infatti una prima tornata di letteratura (risalente fino all'inizio degli anni ottanta) propendeva nettamente per la «superiorità» economica della concertazione neocorporativa (associandole minor disoccupazione e inflazione e più crescita), questa posizione è divenuta l'eccezione nei lavori più recenti, che si distinguono, più o meno accentuatamente, per la comune tendenza a riconoscere il declino del neocorporativismo, a ridimensionare i suoi effetti, o a individuarne di perversi, e a riscoprire le capacità di rendimento del modello alternativo competitivo-pluralista ${ }^{15}$. Naturalmente, le varianti dell'uno o dell'altro autore differiscono non di poco, ma proprio per questo colpisce che vadano tutte nella stessa direzione, tanto più dopo l'iniziale euforia di valutazioni di segno opposto. Se prese insieme, comunque, le informazioni disponibili sottintendono almeno due punti fermi, peraltro crescentemente riconosciuti: il primo è che, contrariamente a quanto sostenuto da qualcuno (Streeck e Schmitter 1985), gli assetti neocorporativi sono (dove ci sono) una determinante del rendimento fra altre, come le dimensioni politico-istituzionali discusse; se e quanto pesino i loro presunti effetti, pertanto, non può essere accertato che nel contesto di queste dimensioni, che, interagendo con quegli assetti, possono potenziarli o inibirli, e così spiegare meglio le eterogenee conclusioni della letteratura; ma - secondo punto - la spiegazione più importante di tanta eterogeneità sembra la variabilità, o l'instabilità, del neocorporativismo stes$\mathrm{so}^{16}$, anch'essa imprevista, o, perlomeno, rivelatasi molto più elevata e accelerata del previsto in parecchi paesi, specie durante l'ultimo decennio. (1989).

15 Streeck (1984); Cawson (1985); Lehmbruch (1985); Schmidt (1988); Schmitter

16 Therborn (1992, 32-33); Gualmini (1997); Huber, Ragin e Stephens (1993, 745); Kriesi (1994, cap. 14). 
Questi limiti hanno spinto qualche autore a invitare ad abbandonare una nozione così problematica (Therborn 1992); ma anche se essa è debole e malformulata, sopprimerla appare troppo drastico, data l'ampia circolazione che continua ad avere. La scelta migliore, quindi, è attestarsi su una via di mezzo. A questo si può provvedere, anzitutto, relativizzando in partenza il neocorporativismo entro un ampio ventaglio di fattori di rendimento; secondo, poiché si conviene che il fenomeno è multidimensionale (Lehmbruch e Schmitter 1982; Lijphart e Crepaz 1991; Huber, Ragin e Stephens 1993), servono evidentemente più indicatori per coglierne appieno la complessità; e, infine, l'uno o l'altro indicatore dovrebbe essere particolarmente sensibile alla proprietà chiave del neocorporativismo (cioè, l'instabilità), così da permettere un tempestivo adeguamento della classificazione dei casi al suo accentuato dinamismo.

Mentre la prima cautela è incorporata nella presente strategia di ricerca (che punta, anzi, sulla batteria più articolata possibile di variabili indipendenti), la tabella 7 mostra come ho provveduto a soddisfare le due restanti: essa, infatti, combina per la prima volta tutti i principali indicatori di neocorporativismo, abbandonando la (cattiva) consuetudine di lavorare solo con quello ritenuto più importante; e utilizza, fra questi indicatori, anche il più trascurato (cioè, la concertazione), che è, però, di sicuro quantomai interessante. La sua rarità, infatti, dipende da una volatilità, o discontinuità, assai maggiore degli altri due, unita alla difficoltà di operazionalizzare pratiche cooperative tipicamente informali. Senza pretendere di risolvere una volta per tutte il secondo problema, ho cercato di trattarlo al meglio altrove (Pappalardo e Mattina 1998) in funzione del primo, cioè per tentare di cogliere la tendenza a mutare dei rapporti Statogruppi (specie i sindacati), come ricordato oggi dominante. Conformemente, la classificazione proposta differisce da altre, anche se, dopotutto, non sistematicamente e con variazioni sostanziali limitate a una minoranza di paesi ${ }^{17}$. Poiché le differenze sono anche effetto della periodizzazione specificamente scelta per questa analisi (1975-1995 circa), mi pare pertanto che la tabella 7 integri e aggiorni, piuttosto che contraddire, il grosso della ricerca precedente e fornisca, al tempo stesso, una base

17 Rispetto al contributo più recente (Lijphart e Crepaz 1991, tab. 1), queste variazioni concernono Belgio, Finlandia, Giappone, Italia, Olanda, Svizzera. 
TAB. 7. Neocorporativismo $1975-1996$

\begin{tabular}{ccc}
\hline Paesi $\begin{array}{c}\text { Tasso di } \\
\text { sindacalizzazione }\end{array}$ & $\begin{array}{c}\text { Centralizzazione } \\
\text { organizzativa e } \\
\text { contrattuale }\end{array}$ \\
\hline
\end{tabular}

\begin{tabular}{llllc} 
Australia & 2 & 4 & 0 & 6 \\
Austria & 2 & 6 & 3 & 11 \\
Belgio & 3 & 4 & 2 & 9 \\
Canada & 2 & 2 & 0 & 4 \\
Danimarca & 3 & 4 & 3 & 10 \\
Finlandia & 3 & $5--$ & 3 & $11-$ \\
Francia & 1 & 3 & 0 & 4 \\
Germania & 2 & $5-$ & 1 & $8--$ \\
Giappone & 1 & 3 & 0 & 4 \\
Gran Bretagna & 2 & 3 & 0 & 5 \\
Italia & 2 & $5-$ & $1+$ & 7 \\
Norvegia & 2 & 4 & 3 & 10 \\
Nuova Zelanda & 2 & $4+$ & 0 & 6 \\
Olanda & 1 & 2 & $2+$ & $7++$ \\
Stati Uniti & 1 & 5 & 0 & 3 \\
Svezia & 3 & 3 & 3 & 11 \\
Svizzera & 1 & & 0 & 4 \\
\hline
\end{tabular}

Nota: Il punteggio del tasso di sindacalizzazione è 3 per valori superiori al $70 \%, 2$ per valori compresi tra il $70 \%$ e il $30 \%, 1$ per valori inferiori al $30 \%$. La centralizzazione organizzativa e contrattuale cumula i due indicatori di Calmfors e Driffill (1988), ciascuno dei quali varia da 0 a 3 ; rispetto alla fonte, sono stati modificati i punteggi di $\mathrm{Da}$ nimarca (da 5- a 4) e Italia (da 3+ a 4-) per tenere conto di recenti sviluppi. Anche alla concertazione, infine, sono attribuiti quattro valori, ossia 0 se trascurabile o inesistente, 1 se marginale e/o intermittente, 2 se più rilevante, 3 se di importanza centrale.

Fonti: Calmfors e Driffill (1988); Ferner e Hyman (1992); Western (1994 e 1995), stime proprie.

più ampia e sicura per valutare una proprietà sistemica che ne ha particolarmente bisogno proprio per il suo apparente dinamismo. Su di essa, quindi, è costruita la graduatoria della tabella 8 , cioè il punto di arrivo di questo lungo paragrafo e, ovviamente, punto di partenza degli altri interrogativi formulati all'inizio del lavoro.

\section{Istituzioni e rendimento}

La tabella 8 richiede come tale pochi commenti. Analogamente ad altre classificazioni, i suoi punteggi sono approssimativi e discutibili, anche se - credo - sostanzialmente giustificati dai dati comparati. Con i lavori schematizzati nella tabella 1 , 
inoltre, ha in comune la salienza assegnata all'alternativa democrazia maggioritaria/democrazia consensuale, che si conferma discriminante quanto (se non più che) in Lijphart e nella successiva letteratura. Il posizionamento dei paesi, però, è spesso diverso, per lo più per effetto dell'arco di tempo, delle variabili, delle operazionalizzazioni e delle tecniche di misurazione prescelte. Rispetto alla graduatoria più autorevole e inclusiva finora disponibile (Lijphart e Crepaz 1991, 245), per esempio, i casi in comune (tutti, meno l'Irlanda) si distribuiscono fra gli estremi dell'Italia e della Gran Bretagna; e mentre l'assegnazione del primato maggioritario a quest'ultima rappresenta un cambiamento marginale (e riflette plausibilmente lo stile decisionale conservatore di quasi tutto il ventennio), lo slittamento italiano al vertice consensuale rappresenta una differenza molto più marcata, ma giustificata da uno stato di esasperato policentrismo, frammentazione e confusa sovrapposizione di poteri deboli e contropoteri forti troppo spesso sfuggito ai, o sottovalutato dai, comparatisti e tutt'altro che superato dalla perdurante transizione (Pappalardo e Mattina 1998). Fra i nostri restanti sistemi maggioritari, poi, figura lo stesso «nocciolo duro» di Lijphart e Crepaz (dall'Australia agli Stati Uniti), con in più il Giappone, la cui posizione di testa si spiega con la struttura unitaria dello stato, il ruolo minore del parlamento e la stabilità del sistema partitico, che i due autori non prendono in considerazione e che contribuiscono a fargli scavalcare parecchie posizioni; ma, più che questo scavalcamento, è il punteggio della Germania che può far discutere, poiché io dò quest'ultima a parità con Francia e Stati Uniti, mentre Schmidt $(1996,172)$ ne fa il modello del policentrismo, dei «governi vincolati», o di «grande coalizione», apparentandola addirittura alla Svizzera. $\mathrm{Da}$ un lato, tuttavia, i suoi indicatori sono singolarmente eterogenei rispetto a quelli usati nella letteratura, oltre che largamente criticabili, come ricordato nel terzo paragrafo. Per entrambi i motivi, dunque, le graduatorie risultanti (della Germania come di altri paesi) non trovano riscontro in quelle di Huber, Ragin e Stephens (1993), Colomer (1995), o degli stessi Lijphart e Crepaz, che sono, semmai, molto più compatibili con la nostra. Come dettagliato altrove (Pappalardo e Mattina 1998), inoltre, stili e contenuti decisionali che Schmidt ritiene documentino pratiche consensuali di «grande coalizione» sono o controversi, o occasionati dalla riunificazione, cioè da un evento senza dubbio eccezionale, comportante molte scelte inevitabilmente una- 
TAB. 8. Democrazie maggioritarie e democrazie consensuali. Punteggi

\begin{tabular}{|c|c|c|c|c|c|c|c|c|}
\hline \multirow[t]{2}{*}{ Paesi } & \multicolumn{2}{|c|}{ Sistema partitico } & \multicolumn{4}{|c|}{ Governi } & \multirow[t]{2}{*}{ Neocorporativismo } & \multirow[t]{2}{*}{ Totale } \\
\hline & $\begin{array}{c}\text { N. effettivo } \\
\text { di partiti }\end{array}$ & $\begin{array}{l}\text { Sta- } \\
\text { bilità }\end{array}$ & Tipo & Durata & esione & Vincoli & & \\
\hline Gran Bretagna & 0 & 0 & 0 & 0 & 1 & 1 & 0 & 2 \\
\hline Australia & 0 & 0 & 0 & 0 & 1 & 3 & 0 & 5 \\
\hline Giappone & 0 & 1 & 0 & 2 & 0 & 2 & 0 & 5 \\
\hline Canada & 0 & 2 & 0 & 1 & 0 & 2 & 0 & 6 \\
\hline Nuova Zelanda & 0 & 2 & 0 & 1 & 2 & 1 & 0 & 6 \\
\hline Francia & 0 & 2 & 1 & 2 & 1 & 0 & 0 & 6 \\
\hline Germania & 0 & 0 & 0 & 0 & 2 & 3 & 1 & 6 \\
\hline Stati Uniti & 0 & 0 & 0 & 1 & 2 & 3 & 0 & 6 \\
\hline Olanda & 2 & 1 & 0 & 1 & 1 & 3 & 1 & 9 \\
\hline Austria & 1 & 1 & 0 & 1 & 2 & 3 & 2 & 10 \\
\hline Svizzera & 2 & 1 & 1 & 1 & 1 & 4 & 0 & 10 \\
\hline Finlandia & 1 & 1 & 1 & 2 & 2 & 2 & 2 & 11 \\
\hline Norvegia & 1 & 1 & 2 & 2 & 1 & 2 & 2 & 11 \\
\hline Svezia & 1 & 1 & 2 & 2 & 2 & 1 & 2 & 11 \\
\hline Danimarca & 1 & 1 & 2 & 2 & 2 & 2 & 2 & 12 \\
\hline Belgio & 2 & 1 & 1 & 2 & 2 & 3 & 1 & 12 \\
\hline Italia & 2 & 2 & 1 & 2 & 2 & 4 & 1 & 14 \\
\hline
\end{tabular}

Nota: (1) Sistema partitico. Numero effettivo di partiti: $\mathrm{N}<3=0,3 \leqslant \mathrm{~N} \leqslant 5=1$, $\mathrm{N}>5=2$. Stabilità (volatilità elettorale): Trend $<0,1=0,0,1 \leqslant$ Trend $\leqslant 0,3=1$, Trend $>0,3=2$. (2) Governi. Tipo: maggioranza monopartitica o minima coalizione vincente $=0$, coalizione sopramisura $=1$, minoranza monopartitica o coalizione di minoranza $=2$. Durata: Superiore a 2.000 gg. $=0$, fra 1.000 e 2.000 gg. $=1$, sotto i 1.000 gg. $=2$. Coesione. Governo: oltre il $40 \%=0$, fra il $20 \%$ e il $40 \%=1$, sotto il $20 \%=2$; Opposizione: sotto il $20 \%=0$, fra il $20 \%$ e il $40 \%=1$, oltre il $40 \%=2$. Vincoli al potere dell'esecutivo: scelta del capo del governo e potere di sfiducia: scelta sottratta al parlamento e sfiducia assente o fortemente vincolata $=0$, scelta sottratta al parlamento e sfiducia moderatamente vincolata, ovvero scelta in parlamento e sfiducia fortemente vincolata $o$ assente $=1$, scelta in parlamento e sfiducia moderatamente o non vincolata $=2$. Bicameralismo: Forte $=2$, debole $=1$, altro $=0$. Decentramento: Federalismo o accentuato regionalismo $=1$, altro $=0$. (3) Neocorporativismo: forte $(10-11$ punti in tab. 7$)$ $=2$, medio $(7-9$ punti $)=1$, debole $o$ assente $=0$.

L'assegnazione di due valori soltanto alla variabile decentramento comporta un peso inferiore a quello degli altri nel punteggio finale. Attribuirle tre valori, tuttavia, non modificherebbe significativamente la graduatoria dei paesi.

nimi, e, appunto per questo, poco o punto rilevanti per valutare la meccanica politica «normale» e a lungo termine del sistema. Tutto sommato, dunque, il posizionamento tedesco nella tabella $8 \mathrm{mi}$ sembra perfettamente difendibile, anche se proprio la riunificazione consiglia prudenza, ma non tanto per ciò che concerne la struttura politico-istituzionale (come crede Schmidt), quanto riguardo al suo rendimento. $\mathrm{Va}$ da sé, infatti, che l'emergenza economica nell'Est del paese rappresenta un poten- 
te fattore di disturbo, unico nell'universo e, in un'ottica comparata, difficile o impossibile da tenere sotto controllo, tanto più che negli ultimi anni non sono più disponibili statistiche EstOvest disaggregate e internazionalmente standardizzate su indicatori come disoccupazione, deficit, spesa pubblica. Poiché queste sono variabili di rendimento cruciali nella successiva analisi, ho pertanto preferito condurre il confronto fra sistemi maggioritari e consensuali scorporandone la Germania e sopprimendo così gli effetti distorcenti della sua temporanea eccezionalità.

Tornando alla tabella 8 , i paesi che restano ricevono punteggi crescentemente «consensuali» e, inclusa la già discussa Italia, figurano tutti dalla stessa parte cui li assegnano Lijphart e Crepaz (1991, 245). Ancora una volta, dunque, c'è una misura di concordanza nell'individuazione dei confini del modello, beninteso insieme a differenze sul posizionamento della sua popolazione. Dati i criteri parzialmente diversi delle due classificazioni, e gli accelerati mutamenti concentrati nei paesi del sottogruppo e non incorporati nei dati «vecchi» dei due autori ${ }^{18}$, peraltro, ci sarebbe da stupirsi del contrario, e ciò che semmai interessa è appunto la linea di demarcazione sostanzialmente comune tracciabile dall'Olanda in poi rispetto ai sistemi maggioritari. Lasciando da parte (per le ragioni dette) la Germania, infatti, questa linea sarà ora utilizzata per una verifica preliminare del rendimento dei due modelli nel loro insieme.

La verifica si può definire preliminare perché rivolta ad accertare se i modelli maggioritario e consensuale, trattati discontinuamente, fanno differenza, piuttosto che quanta differenza si deve a tale o talaltro punteggio della tabella 8 . E mentre a rigore le due questioni si completano a vicenda, solo la prima sarà affrontata qui, e solo nelle linee essenziali. Anche un breve trattamento, però, richiede alcune precisazioni preliminari riguardo all'obiettivo chiave enunciato all'inizio, ossia la spiegazione del rendimento economico delle democrazie in tempi di crisi. La prima precisazione è sollecitata dalla tabella 1 , dalla quale si vede che la, o le, spiegazione/i finora disponibili sono fondate su un numero ridotto di variabili dipendenti: da una a tre, secondo gli autori, che utilizzano naturalmente quelle ritenute più

18 Torno a ricordare che le variabili politico-istituzionali di Lijphart e Crepaz sono costruite su dati 1945-1980, mentre il loro indice di neocorporativismo «si riferisce alla situazione esistente dai tardi anni cinquanta agli anni settanta» $(1991,238)$. 
importanti, ma senza giustificazioni di sorta per ciò che includono o escludono ${ }^{19}$. Per conto mio, invece, mi sono attenuto al criterio meno discutibile sostantivamente e più affidabile metodologicamente, mettendo assieme per la prima volta cinque indicatori di rendimento, più una misura composta di due di essi. Fra questi indicatori figurano, com'è usuale, tasso di crescita, inflazione e disoccupazione (a loro volta sommate nel cosiddetto «indice di miseria»), ma anche deficit e spesa pubblica, quasi mai aggiunte ai precedenti, nonostante l'importanza crescente del loro controllo nelle strategie di «consolidamento fiscale» generalizzatesi in Europa e fuori dopo il secondo shock petrolifero (Oecd, vari anni). Nel complesso, il periodo sottoposto a verifica va dal 1975 al 1994-95 e - seconda precisazione - evita deliberatamente di spingersi più indietro, come fanno, invece, Lijphart (1994a), Crepaz (1996), o Schmidt (1996). Questi autori si servono infatti di più lunghe serie di dati, fornite sotto forma di medie di variabili (indipendenti e dipendenti) calcolate a partire dall'inizio degli anni sessanta (tab. 1). E mentre credo di aver dimostrato che il dinamismo politico-istituzionale di molti paesi viene così indebitamente distorto, $\mathrm{i}$ problemi - teorici ed empirici - si moltiplicano quando si cerca di verificarne il rendimento senza discriminare fra periodi di crisi e periodi di prosperità (come, appunto, gli anni sessanta).

Da un punto di vista teorico, per esempio, c'è da chiedersi quale sia il contesto cruciale per valutare l'impatto delle istituzioni; e la risposta - condivisa da una crescente letteratura - è che, «mentre le differenze strutturali fra sistemi di governo possono contare poco o nulla in tempi economicamente tranquilli, il loro effetto può diventare davvero significativo e influente allorché ci si misura con serie difficoltà $»^{20}$. Ergo, serie indiscriminatamente lunghe non vuol dire serie capaci di ottimizzare la verifica, ma, più probabilmente, il contrario. Per intendersi, mescolando il «consenso keynesiano» dei tempi d'abbondanza alle controversie monetariste suscitate dalla stagflazione, le scelte «facili», espansive e convergenti pre-shocks petroliferi e quelle

19 Mi riferisco al trattamento econometrico di dati quantitativi e, dunque, escludo i molto più articolati contributi del volume di Weaver e Rockman e l'indice di inefficienza dei servizi sociali di Siniscalco e Tabellini, chẹ è calcolato su atteggiamenti (grado di insoddisfazione dei cittadini).

20 Borrelli e Royed (1995, 227); Roubini e Sachs (1989a, 925); Keman, Paloheimo e Whiteley (1987); Weir e Skocpol (1983). 
«difficili», restrittive e più nettamente differenziate successive, i dati non possono che elidersi a vicenda, confondendo in un'artificiosa «capacità media» eventuali divaricazioni in condizioni di «risposta a sfida»; e, conformemente, il ricercatore dovrà accontentarsi di risultati econometrici nulli o molto deludenti: come, per esempio, i coefficienti di Lijphart (1994a, 11), non significativi e piccoli abbastanza da suggerire solo «un rendimento un po' migliore delle democrazie più consensuali» su inflazione e disoccupazione (ma non sulla crescita economica); oppure, quelli francamente irrilevanti di Crepaz (1996, 97-98) e Schmidt (1996, 176), che fanno differenze di segno, ma per spostamenti della retta di regressione dallo 0,05 allo 0,1 !

Anche l'aspettativa di effetti empirici più robusti, dunque, consiglia di distinguere gli stati di «normalità», governabili con risorse modeste presumibilmente sufficienti per politiche di routine, dalle sfide ambientali che sollecitino la massima mobilitazione di cui un dato sistema è capace, dando così il dovuto risalto a tutto il potenziale di rendimento - poco o tanto che sia - delle sue istituzioni. Ma un'ultima precisazione è che questo potenziale non è adeguatamente espresso dai valori medi delle variabili dipendenti sui quali lavorano gli usuali modelli econometrici (Goldberger 1968). Ancorché limitati correttamente ai periodi di crisi, infatti, valori medi tendono per definizione a livellare l'instabilità, o variabilità, delle serie temporali e, di conseguenza, a minimizzare, o sottostimare, una proprietà centrale del policy-making, ossia la gradualità, la lentezza, i ripensamenti o fallimenti ed, eventualmente, la bassa sistematicità e coerenza che accompagnano l'azione pubblica, specie quando si tratta di compiere difficili scelte restrittive di fronte a shocks improvvisi e di grande portata. Poiché la letteratura qualitativa documenta un'impressionante concentrazione di tutti questi elementi nel periodo in esame ${ }^{21}$, la soluzione più logica e informativa è, ovviamente, tentare di trasferirli nell'analisi quantitativa, piuttosto che seguire il discutibile uso di procedere su binari separati. Per questo, in concreto, è necessario sottoscrivere il sottinteso di quanto ora detto, cioè che il potenziale di rendimento delle istituzioni richiede tempo per entrare a regime, o, per ripetersi, prove ed errori, processi di apprendimento spesso tortuosi, aggiustamenti e riaggiustamenti anche contraddittori. Conforme(1993).

21 Oecd (vari anni); Peele (1988); Pierson (1994 e 1996); Weaver e Rockman 
mente, gli eventuali effetti si devono supporre dinamici, variabili e, lungi dall'essere livellati nelle medie, questa variabilità va valorizzata come espressione del «reale» comportamento dell'explanans. In altre parole, se il tempo è così importante per il rendimento come corroborato dalla ricerca qualitativa, del tempo bisogna tenere il dovuto conto, e in termini diacronici devono essere poste due domande cruciali per un'adeguata verifica: quali tendenze (a crescente, decrescente, stagnante rendimento) sono associate al modello maggioritario e al modello consensuale? E quanto rapide o pronunciate?

Queste domande, peraltro, non esauriscono l'argomento, né escludono un'ispezione delle medie, anch'essa importante, ma si vedrà - logicamente successiva. Intanto, la tabella 9 riporta, appunto, le tendenze (o regressioni sul tempo) di inflazione, disoccupazione, indice di miseria, spesa pubblica, deficit e crescita del PIL nei due sottogruppi di paesi scelti come unità di analisi (e, per conto suo, il difficile caso tedesco). Come si vede, i dati suggeriscono un generale, più o meno accentuato, dinamismo di tutte le variabili in tutti, o parte dei, periodi considerati. Naturalmente, il fatto è tutt'altro che imprevisto, riflette semplicemente gli shocks subiti dalle democrazie industriali e gli sforzi dei loro governanti di rispondervi ben documentati da un'enorme letteratura. $\mathrm{Ma}$, a misura che le tendenze registrate dalla tabella riflettono questi sforzi, la constatazione più importante è che essi non hanno avuto lo stesso successo, o lo stesso rendimento. Cominciamo, infatti, dalla lotta all'inflazione, che è stata la, o una delle, priorità di tutte le politiche anticrisi del ventennio; conformemente, la variabile mostra il più rapido andamento discendente fra tutti gli indicatori, oltre che il più sistematico attraverso i periodi; e questo fa risaltare la differenza che resta, cioè il distacco dato dai sistemi maggioritari ai sistemi consensuali, sia nel ventennio che nei due decenni, e specialmente nel 1985-1995.

La successiva variabile non modifica la conclusione, poiché trends ascendenti da disoccupazione di massa si registrano costantemente dove meno lo si sarebbe aspettato (cioè, fra i sistemi consensuali per lo più socialdemocratici), mentre i conservatori predominanti dall'altra parte esordiscono peggio nel 19751985, ma decelerano di tre quarti dal 1985 e finiscono con incrementi annui nel ventennio quasi dimezzati $(+0,148)$ rispetto al modello concorrente $(+0,238)$. I dati, quindi, documentano un ripiegamento storico della più imperativa priorità socialde- 
TAB. 9. Istituzioni ed economia - Trends

\begin{tabular}{|c|c|c|c|c|c|c|c|c|c|}
\hline & \multicolumn{3}{|c|}{ Sistemi maggioritari } & \multicolumn{3}{|c|}{ Sistemi consensuali } & \multicolumn{3}{|c|}{ Germania } \\
\hline & $1975-1985$ & $1985-1995$ & $1975-1995$ & $1975-1985$ & $1985-1995$ & $1975-1995$ & $1975-1985$ & $1985-1995$ & $1975-1995$ \\
\hline Disoccupazione & 0,425 & 0,104 & 0,148 & 0,351 & 0,348 & 0,238 & 0,447 & 0,113 & 0,136 \\
\hline Inflazione & $-0,680$ & $-0,442$ & $-0,555$ & $-0,398$ & $-0,173$ & $-0,426$ & $-0,196$ & $-0,256$ & $-0,125$ \\
\hline Indice di miseria & $-0,297$ & $-0,271$ & $-0,4$ & $-0,048$ & 0,176 & $-0,201$ & 0,251 & 0,263 & 0,015 \\
\hline Spesa pubblica & 0,297 & 0,304 & 0,296 & 1,013 & 0,608 & 0,571 & $-0,017$ & 0,325 & $-0,013$ \\
\hline Deficit & $-0,088$ & $-0,281$ & $-0,026$ & $-0,249$ & $-0,454$ & $-0,104$ & $-0,223$ & $-0,219$ & 0,072 \\
\hline Crescita del PIL & 0,151 & $-0,193$ & $-0,009$ & 0,070 & $-0,229$ & $-0,034$ & $-0,072$ & $-0,038$ & 0,051 \\
\hline
\end{tabular}

Nota: Sistemi maggioritari: punteggi da 2 a 6 in tab.; sistemi consensuali: punteggi da 9 a 14. Trends positivi indicano aumenti di disoccupazione, inflazione, indice di miseria, spesa pubblica e crescita. Trends negativi indicano aumenti del deficit. Disoccupazione, inflazione e indice di miseria sono stati calcolati fino al 1995; le restanti variabili, e tutti i dati tedeschi, fino al 1994. Tasso di disoccupazione non standardizzato per Austria, Danimarca, Svizzera, standardizzato in tutti gli altri casi e disponibile solo dal 1986 per la Nuova Zelanda. Deficit e spesa pubblica non disponibili per Nuova Zelanda e Svizzera.

Fonte: OECD (vari anni), calcoli propri. 
TAB. 10. Istituzioni ed economia - Medie

\begin{tabular}{|c|c|c|c|c|c|c|c|c|c|c|c|c|c|c|c|c|c|c|}
\hline & \multicolumn{3}{|c|}{ Disoccupazione } & \multicolumn{3}{|c|}{ Inflazione } & \multicolumn{3}{|c|}{ Indice di miseria } & \multicolumn{3}{|c|}{ Spesa pubblica } & \multicolumn{3}{|c|}{ Deficit } & \multicolumn{3}{|c|}{ Crescita del PIL } \\
\hline & I & II & III & I & II & III & I & II & III & I & II & III & I & II & III & I & II & III \\
\hline $1975-1977$ & 5,3 & 3,7 & 3,6 & 12 & 10,2 & 4,8 & 16,7 & 13,8 & 8,4 & 36,1 & 46,0 & 47,8 & $-2,7$ & $-1,6$ & $-3,8$ & 2,3 & 1,8 & 2,2 \\
\hline $1978-1980$ & 5,6 & 4,4 & 3,2 & 10,5 & 8,0 & 4,1 & 15,3 & 12,4 & 7,3 & 36,5 & 50,3 & 47,5 & $-2,4$ & $-2,7$ & $-2,6$ & 2,3 & 3,1 & 2,7 \\
\hline $1981-1983$ & 8,0 & 6,1 & 5,9 & 8,9 & 8,9 & 5 & 15,8 & 15,1 & 10,9 & 39,6 & 54,1 & 48,5 & $-3,1$ & $-4,9$ & $-3,2$ & 1,9 & 1,0 & 0,3 \\
\hline $1984-1986$ & 8,1 & 6,2 & 6,9 & 5,3 & 4,8 & 1,5 & 12,7 & 11,1 & 8,4 & 40,4 & 53,8 & 46,9 & $-3,2$ & $-2,6$ & $-1,5$ & 3,8 & 2,9 & 3,4 \\
\hline $1987-1989$ & 6,8 & 5,7 & 6 & 5,1 & 3,7 & 1,4 & 11,8 & 9,5 & 7,4 & 38,4 & 53,4 & 45,9 & $-0,7$ & $-1,7$ & $-1,3$ & 3,5 & 2,7 & 2,9 \\
\hline $1990-1992$ & 7,9 & 6,2 & 4,5 & 3,9 & 4,2 & 3,4 & 11,8 & 10,4 & 7,9 & 40,5 & 55,5 & 47,2 & $-2,5$ & $-3,3$ & $-2,8$ & 0,6 & 1,1 & 4,3 \\
\hline $1993-1995$ & 8,3 & 8,9 & 8,2 & 2 & 2,5 & 3 & 10,4 & 11,4 & 11,2 & 42,0 & 57,6 & 49,3 & $-4,4$ & $-5,2$ & $-3,1$ & 2,6 & 1,7 & 1,2 \\
\hline
\end{tabular}

Nota: I: sistemi maggioritari; II: sistemi consensuali; III: Germania

Fonti: OECD (vari anni), calcoli propri. 
mocratica, enfatizzato dal rapido recupero dei governi meno impegnati programmaticamente sul fronte occupazionale. Come dire che la volontà politica non basta, plausibilmente perché i partiti - «onnipresenti, ma non onnipotenti» - devono disporre di adeguate capacità istituzionali per tradurla in pratica. E va da sé che questo è confermato dalla terza variabile (indice di miseria), cioè dalla somma di inflazione e disoccupazione. Come tale, anzi, essa non può che accentuare il vantaggio dei sistemi maggioritari, che, nel complesso del periodo, ottengono risultati doppiamente migliori, riducendo l'indice dello 0,4 all'anno contro lo 0,2; mantengono lo stesso distacco nel 19751985, evidentemente per effetto della maggior efficacia delle loro politiche antinflazionistiche; e lo massimizzano nel 19851995, quando il loro decremento dello 0,27 annuo si contrappone all'aumento $(+0,176)$ della «miseria» dei sistemi consensuali.

Per di più, il rendimento comparativamente mediocre di questi ultimi sembrerebbe essere stato costoso, o, comunque, molto più costoso di quello altrui. Nella casella relativa alla spesa pubblica, infatti, non è il trend continuamente ascendente che stupisce, trattandosi di un effetto universale noto della crisi (Roubini e Sachs 1989b); molto più impressionanti sono i dati da finanze fuori controllo del sottogruppo consensuale, specie nel 1975-1985, ma anche nel periodo successivo e nell'intero ventennio. Il +0,57 annuo 1975-1994, infatti, è un record in sé, oltre che il doppio del dato maggioritario; e se una parte della differenza conferma la tradizionale maggior propensione socialdemocratica a spendere, la sua componente più importante è comprovatamente «involontaria», cioè dovuta a lassismo politico, o dettata dalla necessità di «guadagnare tempo» in un contesto di vincoli forti e tenacemente resistenti all'innovazione politica ${ }^{22}$. Solo dal 1993-1994 (e quindi fuori dalla portata dei dati), si è generalizzato uno sforzo di riaggiustamento (Oecd 1997), che non autorizza, però, conclusioni definitive su un ritrovato «decisionismo», o una recuperata efficienza istituzionale. I deficit costantemente crescenti, specie nel 1985-1995, infatti, suggeriscono piuttosto una svolta imposta in gran parte dall'emergenza in cui la spesa fuori controllo ha finito col gettare $i$

22 La scelta (o non scelta) di guadagnare tempo è stata particolarmente pronunciata nei paesi scandinavi (Pekkarinen 1992). Per maggiori dettagli, vedi Pappalardo e Mattina (1998). 
conti pubblici. Ma, come che sia, anche con questo dato la tabella fornisce un inequivocabile verdetto comparato, promuovendo di nuovo, attraverso tutti i periodi, il rendimento maggioritario su quello consensuale.

Poco o punto discriminanti, viceversa, sono gli andamenti del PIL, cioè della variabile (crescita) più colpita dalla crisi e più decisiva per il suo superamento, ma, a quanto pare, più resistente agli sforzi di tutti i governi di ripristinarla. La regressione «piatta» sul ventennio, infatti, è il dato al riguardo più eloquente e deludente, anche se - come tale - non toglie (né aggiunge) nulla alle conclusioni tratte. Qualcosa di più, però, si può dire esaminando i dati in una forma diversa, per affrontare una questione generale che i trends, con tutti i loro meriti, lasciano inevasa. La questione è che, scontato lo sforzo più rapido e più riuscito dei sistemi maggioritari di recuperare rendimento, può essere interessante evidenziarne il livello e compararlo a quello dei sistemi consensuali. Per questo, ai trends vanno sostituiti i valori medi, che ho riportato nella tabella 10 , accorpati per trienni. Essi documentano, per cominciare, che il generale successo delle politiche antinflazionistiche è culminato per tutti i paesi nei minimi storici del 1993-1995; ma, altrettanto evidentemente, quelli maggioritari sono partiti peggio e finiti meglio, scavalcando i consensuali dall'inizio degli anni novanta. Conformemente a quanto sostenuto da molti, d'altro canto, meno inflazione si è accompagnata a più disoccupazione, seppure con andamenti differenziati nel tempo: a un inequivocabile e costoso trade off fra le due fino a metà anni ottanta, infatti, è seguita una sostanziale stabilizzazione della seconda nel sottogruppo maggioritario, mentre quello consensuale ha tenuto duro fino al 1992, ma accusa subito dopo un aumento repentino del $25 \%$ che lo porta a scavalcare l'altro alla fine del periodo; e se, da un lato, lo scavalcamento si può dire marginale, non lo è, dall'altro, una progressione verso la disoccupazione di massa dal 3,7 nel 1975-1977 all'8,9 dell'ultimo triennio; né l'effetto che, cumulandosi all'inflazione, essa ha sull'indice di miseria, ridottosi di oltre un terzo nell'aggregato maggioritario (dal $15,6$ al 10,2$)$ e di appena il $17 \%$ fra i paesi consensuali.

Con 1 ' $11,4 \%$ contro il $10,4 \%$, dunque, questi ultimi si confermano in ritardo, per di più nel contesto di squilibri finanziari davvero preoccupanti: i trends già ricordati della spesa pubblica, infatti, equivalgono a 12 punti circa di aumento dal 1975 (contro i sei dei maggioritari), o, se si vuole, a una divaricazione 
fra i due sottogruppi di quasi il $16 \%$; e se una maggior propensione consensuale a compensare più spese con più pressione fiscale aveva in passato tamponato le falle di bilancio ${ }^{23}$, $i$ dati più recenti al riguardo mostrano che neanche questa è più una soluzione efficace o praticabile: da un deficit trascurabile, e pari alla metà di quello maggioritario, il passaggio al $-5,2$ (contro il $-4,4 \%$ ) segnala, infatti, il culmine di un'altalena di valori diversi, ma sempre negativi e, in cinque trienni su sette, peggiori del termine di confronto. Né diverso, infine, il verdetto sulla crescita, che i trends davano ugualmente «piatta» in tutti i paesi; ma, se esaminata per livelli, è stata mediamente più accelerata nei sistemi maggioritari, anche questa volta per cinque trienni.

Questa prima lettura dei dati autorizza perlomeno un bilancio minimo, fatto di tre considerazioni cautelative e un risultato certo. La prima considerazione riguarda il trattamento dicotomico dei casi, che, ovviamente, impedisce di individuare quale precisa combinazione di proprietà (maggioritarie e consensuali) sia responsabile di quanto rendimento. Rispetto a questo (che necessiterebbe un'analisi econometrica da rimandare ad altra sede), mi sono limitato ad un'esplorazione preliminare, a quanto pare promettente perché rivela che i due modelli effettivamente differiscono e, in tal senso, apre ragionevolmente la via ai successivi interrogativi. Tanto l'esplorazione, quanto i futuri approfondimenti, comunque, dovrebbero essere accompagnati dalle altre due cautele, a cominciare dalla consapevolezza che la loro portata non può che essere time specific, o vincolata dal tempo. Anche al riguardo, del resto, si è detto più sopra che il tempo non è neutrale, poiché attraverso di esso si dispiegano mutamenti istituzionali (cioè, delle variabili indipendenti), contestuali (con l'alternarsi di periodi di crisi e periodi di prosperità) e, a fortiori, di rendimento. A questa stregua, dunque, non si dovrebbe mai perdere di vista la natura relativa di qualunque classificazione e delle conclusioni che se ne possono trarre, né la possibilità che - a classificazione anche invariata delle loro configurazioni istituzionali - alcuni paesi apprendano dai successi di altri e ne imitino deliberatamente le strategie, riuscendo a correggere - seppure in ritardo - $\mathrm{i}$ loro eventuali squilibri. Per qualcuno - o per tutti - questi motivi, insomma, nessun complesso politico-istituzionale va considerato dato una volta

23 Ancora una volta, sono i paesi scandinavi a guidare questa tendenza, specialmente la Svezia. Borrelli e Royed (1995, 238-243); Steinmo (1989). 
per tutte, o vincolante al punto da condannare al fallimento o da garantire il rendimento. Tanto è vero, che una lettura caso per caso (Pappalardo e Mattina 1998) rivela più o meno pronunciate tendenze alla convergenza, essenzialmente per effetto di un recupero di rendimento dei sistemi consensuali, sensibile soprattutto sul finire del periodo esaminato. Senza eliminarle, l'apprendimento e l'imitazione stanno così riducendo le differenze, e tutto sta a vedere fino a che punto la riduzione potrà spingersi senza una solida strumentazione istituzionale.

C'è una circostanza, però, che potrebbe stabilizzare la convergenza e far passare la strumentazione dei singoli paesi in secondo piano. Come tale, essa suggerisce la terza considerazione cautelativa, particolarmente importante perché in grado di mettere in dubbio il futuro di un'intera tradizione di ricerche comparate. Un requisito chiave di queste ricerche (inclusa la presente) è, infatti, «l'indipendenza dei casi» (Lijphart 1975), o, per intendersi, una sovranità e un'autonomia sufficiente delle istituzioni nazionali da permettere di utilizzare le loro variazioni per spiegare quelle del rendimento. $\mathrm{Ma}$, come altre proprietà, l'indipendenza delle istituzioni non è data o immutabile e da tempo, anzi, è - da più parti e con varie giustificazioni - qualificata, relativizzata, o contestata tout court. Senza entrare nei dettagli del dibattito, la conclusione più ragionevole e documentata che se ne può trarre sta - come spesso avviene - in mezzo: nonostante un gran parlare di policy convergence, di comparazione per settori o regioni transnazionali, o - addirittura - di un unico «sistema mondo» da sostituire all'unità di ricerca tradizionale (cioè, lo Stato-nazione), quest'ultimo si è infatti rivelato resistente e indipendente quanto basta da impulsi esterni di qualsiasi natura e origine da meritare il ruolo privilegiato finora accordatogli dalla gran maggioranza dei ricercato$\mathrm{ri}^{24}$; correttamente, dunque, ho a mia volta messo lo Stato-nazione, $o$, più precisamente, alcuni suoi attributi strutturali, al centro del lavoro; e, tuttavia, questo non impedisce di riconoscere che eventi recentissimi stanno mettendo in moto processi

24 Alquanto paradossalmente, questa è la conclusione di uno dei più ambiziosi progetti di comparazione per settori, secondo il quale «quasi tutti i regimi (di regolazione) internazionali si sovrappongono a forti ed eterogenei regimi nazionali che non sembrano destinati a scomparire tanto presto» (Hollingsworth e Streeck 1994, 290). Analoga l'opinione di Bennett (1991) e Van Deth (1995), che discutono inoltre i complessi problemi concettuali e metodologici associati allo spostamento del centro di gravità della ricerca comparata. 
nuovi e rapidi che ne annunciano il superamento, o, perlomeno, un ridimensionamento significativo; mi riferisco, ovviamente, all'accelerazione dell'integrazione europea dovuta al Trattato di Maastricht e alle scadenze del 1998, con le loro conseguenze per i paesi aderenti, ma anche per alcuni outsiders comunque indirettamente coinvolti (Gran Bretagna, Norvegia); senza dubbio, infatti, le conseguenze sono sempre più vincolanti per le politiche economiche e di riequilibrio finanziario nazionali e mettono in risalto il vero motore dello sforzo di alcuni sistemi consensuali di recuperare i loro ritardi; paesi come Italia, Danimarca, Finlandia, Svezia, per esempio, stanno ora procedendo a questo recupero e - si è detto - dimostrano così di aver appreso dai, e imitato $i$, successi maggioritari; ma apprendimento e imitazione appaiono sempre meno volontari e sempre più forzati da pressioni e obiettivi sovranazionali, peraltro a loro volta meno prescelti deliberatamente che imposti da tendenze e sfide mondiali che richiedono risposte su scala europea; conseguentemente, future analisi dovranno probabilmente porsi su questa scala e procedere a comparare blocchi politico-economici continentali (Europa, Nord-America, Giappone+Sud-Est asiatico) per garantirsi l'indipendenza dei casi e spiegazioni valide; ma per chi lavora sul passato - incluso il passato prossimo - ripeto che gli Stati nazionali rimangono il contesto più appropriato per mettere a punto indicatori, formulare ipotesi e procedere a verificarle.

Siamo così all'ultimo punto da discutere, cioè alle conseguenze della verifica per gli argomenti a favore o contro le democrazie maggioritarie e le democrazie consensuali. Se - come ho documentato - il rendimento delle prime ha prevalso sul rendimento delle seconde, la conclusione di questa analisi è chiaramente in controtendenza rispetto ad autori come Lijphart (1994a) e Crepaz (1996), ma d'accordo con altri (Roubini e Sachs 1989a e 1989b; Siniscalco e Tabellini 1993). Fra disaccordo e accordo, naturalmente, c'è uno spartiacque cronologico, cioè la scelta di accorpare tempi di prosperità e di crisi (Lijphart e Crepaz 1991), ovvero di isolare questi ultimi, come io stesso ho ritenuto più plausibile teoricamente e più fruttuoso empiricamente. Ma se questa (ed altre) differenze di impostazione condizionano i risultati ottenuti, spiegarli è un'altra questione, che richiede di tornare al dibattito sui modelli di democrazia sintetizzato nel terzo paragrafo. L'aspetto centrale di questo dibattito è colto da Lijphart $(1994 a, 12)$ con l'osserva- 
zione - mutuata da Finer (1975) ed altri - che «l'alternanza al governo, caratteristica del modello maggioritario, può comportare cambiamenti di politica economica troppo frequenti e repentini, mentre molti esperti sono venuti alla conclusione che il successo macroeconomico richieda non tanto la mano forte, quanto una guida continua». Per Lijphart, e più accentuatamente per Crepaz (1996, 94-96), la «guida continua» è, a sua volta, propria del modello consensuale e, poiché «ciò che conta sono la stabilità e la prevedibilità delle politiche pubbliche», sarebbe così spiegato il rendimento (debolmente) migliore che gli attribuiscono i due autori. Ma la spiegazione è, a ben guardare, doppiamente malposta, fallace nella premessa non meno che nelle conclusioni. Dai dati discussi, infatti, è il modello maggioritario ad uscire comparativamente bene; e al suo rendimento possono aver contribuito precisamente la stabilità e la continuità della guida politica dei paesi interessati, sicuramente maggiori - non minori - che nei regimi consensuali: inequivocabili, al riguardo, sono i records di durata dei governi in Australia, Canada, Gran Bretagna, Nuova Zelanda, Stati Uniti, risultanti dalla tabella 4, ma anche dai dati di Lijphart (1984, 78-85); è così confermato quanto a suo tempo osservava Sartori (1982, 193199), ossia che non l'alternanza, ma lunghi cicli di egemonia di una parte sull'altra, contraddistinguono il bipartitismo; e - è vero - l'egemonia (o durata) non garantisce il rendimento (Lijphart 1994a, 3-4); ma che dire allora della combinazione consensuale di governi meno durevoli e meno coesivi documentata dalle tabelle 4 e 5 ? Come minimo, essa è proporzionatamente controproducente e, quand'anche giudicata non significativa, indica senz'altro che l'alternanza si trova dal lato opposto a quello in cui la cercano Lijphart e Crepaz.

A difesa, peraltro, questi ultimi potrebbero sostenere (anzi, effettivamente sostengono) che nei sistemi multipartitici, di solito, «uno o due membri minori della coalizione sono sostituiti con altri» (Crepaz 1996, 95-96), cioè non si verifica più di una semirotazione al potere, mentre «cambiamenti di politiche pubbliche su più larga scala» avvengono allorché «le responsabilità di governo passano regolarmente di mano fra due partiti su posizioni molto diverse... e la politica diviene suscettibile di prendere un andamento convulso», in quanto il nuovo arrivato dedicherà «energie, risorse e tempo a rovesciare le decisioni del governo precedente, dando luogo a prematuri altolà e false partenze». La conclusione, dunque, è nuovamente a favore di una 
maggiore «continuità e prevedibilità» d'azione delle coalizioni con «uno o due membri in pianta stabile», e sarebbe ragionevole se non fosse per due problemi. Il primo, ma non il maggiore, è che diversi sistemi consensuali (Belgio, Danimarca, Norvegia, Olanda, Svezia) hanno sperimentato dalla metà degli anni settanta rotazioni al potere totali, o quasi totali, più frequenti dei maggioritari. Il secondo, ben più decisivo, è che la precedente descrizione delle conseguenze dell'alternanza è infondata, per non dire caricaturale. Quali che siano le posizioni elettorali dei competitori nei sistemi maggioritari, infatti, la loro trasmissione nelle politiche economiche non avviene affatto per «rovesciamenti» - tantomeno su larga scala o repentini - di quanto realizzato dal predecessore. Nonostante alcune voci discordanti, infatti, proprio il contrario è da attendersi da una massiccia tradizione teorica che associa il bipartitismo alla competizione centripeta, alla massimizzazione del voto dell'elettore mediano, alla rappresentanza dell' «interesse generale» e, in breve, a condotte improntate a moderazione ed equilibrio ${ }^{25}$. $\mathrm{Ma}$, teorie a parte, ho già ricordato che aggiustamenti incrementali e riforme selettive, parziali, pragmaticamente graduate alle resistenze, sono regole chiave del policy making, alle quali non si sottraggono campioni di cambiamento e di decisionismo come i governi Reagan e Thatcher, o, sul fronte opposto, i socialisti france$\mathrm{si}^{26}$. Come dire che, anche quando c'è, l'alternanza fa differenze senza escludere fondamentali continuità, e le differenze stesse maturano di solito con sufficiente lentezza da prevenire o minimizzare eventuali reazioni ostili. Cade così, o è confinata alle eccezioni, l'ultima imputazione alle istituzioni maggioritarie, cioè il presunto rischio di instabilità associato a decisioni prese «in contrasto con le preferenze di importanti settori della società» (Lijphart 1994a, 12). Eccezionale è, infatti, l'esempio più spesso portato a sostegno (la poll tax introdotta dal governo Thatcher), frutto, peraltro, di un errore di calcolo riparabile - e riparato - con normalissimi strumenti di routine parlamentare (ritiro del provvedimento e sostituzione del primo ministro) e senza conseguenze elettorali per i! partito al potere. Non sembra proprio, dunque, che il maggioritario «tenda a far ingurgitare forzatamente politiche indesiderate» (Robertson 1984, 217), o, perlomeno, così indesiderate da meritare drastiche pu-

25 Downs (1957); Sartori (1976); Almond e Powell (1978); Olson (1986).

26 Vedi la bibliografia alla nota 14 e, sui socialisti francesi, Pappalardo (1991). 
nizioni. Anzi, mai come nell'ultimo ventennio i governi di Australia, Gran Bretagna, Stati Uniti o Nuova Zelanda sono stati premiati con ripetute conferme, o con records di pace sociale ineguagliati dai sistemi consensuali ${ }^{27}$. E allora?

Allora, il paradosso (ma non tanto) è che la straordinaria stabilità maggioritaria sottintende più (o, certo, non meno) sostegno della controparte consensuale. Con la stabilità e il sostegno (o, se si preferisce, la tolleranza) della società, hanno potuto essere sfruttate appieno le «virtù» incontestate del modello Westminster: tempestività di azione, capacità di innovare, imporre sacrifici, fissare priorità, allocare selettivamente risorse, subordinare obiettivi confliggenti e porre in essere le scelte fatte (Weaver e Rockman 1993, 454-457). A sua volta, questo può aver richiesto una misura di «mano forte»; ma graduata, selettiva e solo lentamente cumulativa come è stata, i suoi costi sono stati bene o male assorbiti, e i suoi benefici crescentemente riconosciuti. Tanto è vero, che anche le rotazioni al potere degli anni novanta (in Australia, negli Stati Uniti, in Gran Bretagna, $o$ in Francia) danno inequivocabili segni di continuità, nello stile decisionale come nelle scelte di politica economica; e perfino i sistemi consensuali sono impegnati in uno sforzo di convergenza, dettato in parte - si è detto - dai vincoli internazionali, ma comunque associato al ridimensionamento di storici «punti di veto», pratiche unanimistiche, compromessi inclusivi: esemplari, al riguardo, i paesi scandinavi, che hanno cessato così di attardarsi a «guadagnare tempo» (Pekkarinen 1992) e sono passati all'attacco di squilibri troppo a lungo lasciati accumulare.

Se l'imitazione è l'ennesimo, e conclusivo, segno di successo, proprio la svolta scandinava, però, ne ricorda i limiti, imponendo con particolare forza all'attenzione il classico dilemma delle scelte pubbliche: meno costi decisionali in cambio di più costi esterni $\mathrm{e}$, in concreto, più disoccupazione, meno protezione sociale, più disuguaglianze ed emarginazione. Questo dilemma, familiare ai sistemi maggioritari, è ora reso più drammatico dal trasferimento a paesi che se ne ritenevano immuni, poiché l'ovvia inferenza è che non esiste soluzione capace di massimizzare simultaneamente dimensioni cruciali dell'«interesse genera-

27 Nelle relazioni industriali, in particolare, $i$ quattro paesi hanno toccato minimi storici dei tassi di sciopero (stabili o in salita, invece, in diversi paesi consensuali). $\mathrm{Ma}$ anche indicatori come manifestazioni, disordini, delitti politici hanno fatto progressi, rafforzando il loro tradizionale primato nel mantenimento di «legge e ordine». 
le»: a quanto pare, quel che si guadagna in efficienza con istituzioni e stili maggioritari si paga con la solidarietà e l'equità, a loro volta più centrali quando prevalgono pratiche consensuali. Con la definizione di rendimento adottata, solo la prima dimensione mi ha occupato in questa sede; ma solidarietà ed equità hanno altrettanto titolo a formarne un'altra non meno importante, né meno necessaria, per un bilancio veramente definitivo dei meriti o demeriti dei due modelli di democrazia.

\section{Riferimenti bibliografici}

Almond, G. e G.B. Powell (1978²), Comparative Politics, Boston, Little Brown.

Bardi, L. (1996), Change in the Italian Party System, in «Italian Politics and Society», n. 46, autunno, pp. 9-22.

Bartolini, S. e R. D'Alimonte (1995), Maggioritario ma non troppo, Bologna, Il Mulino.

Bartolini, S. e P. Mair (1990), Identity, Competition and Electoral Availability. The Stabilization of European Electorates 1885-1985, London, Cambridge University Press.

Bennett, C. J. (1991), Review Article: What Is Policy Convergence and What Causes It?, in «British Journal of Political Science», 21, pp. 215-233.

Berger S. (a cura di) (1981), Organizing Interests in Western Europe, London, Cambridge University Press; trad. it., L'organizzazione degli interessi nell'Europa occidentale, Bologna, Il Mulino, 1983.

Blais, A., D. Blake e S. Dion (1993), Do Parties Make a Difference? Parties and the Size of Government in Liberal Democracies, in «American Journal of Political Science», 37, pp. 40-62.

Borrelli, S.A. e T.J. Royed (1995), Government 'Strength' and Budget Deficits in Advanced Democracies, in «European Journal of Political Research», 28, pp. 225-260.

Buchanan, J.M. e G. Tullock (1962), The Calculus of Consent, Ann Arbor, University of Michigan Press.

Calmfors, L. e J. Driffill (1988), Bargaining Structure, Corporatism and Macroeconomic Performance, in «Economic Policy», 6, pp. 14-61.

Cameron, D.R. (1984), Social Democracy, Corporatism and Labor Quiescence, in Goldthorpe (1984), pp. 143-178.

Castles, F.G. (1982), The Impact of Parties on Public Expenditures, in F.G. Castles (a cura di), The Impact of Parties. Politics and Policies in Democratic Capitalist States, London, Sage, pp. 21-96.

Castles, F.G., F. Lehner e M.G. Schmidt (a cura di) (1988), Managing Mixed Economies, Berlin, De Gruyter.

Castles, F.G. e R.D. McKinlay (1979), Does Politics Matter: An Analy- 
sis of the Public Welfare Commitment in Advanced Democratic States, in «European Journal of Political Research», 7, pp. 169-186.

Cawson, A. (1985), Introduction: Varieties of Corporatism. The Importance of Meso-Level Interest Representation, in A. Cawson (a cura di), Organized Interests and the State, London, Sage, pp. 1-22.

Colomer, J.M. (1995), Introduzione, in J.M. Colomer (a cura di), La politica in Europa, Bari, Laterza.

Crepaz, M.M.L. (1992), Corporatism in Decline? An Empirical Analysis of the Impact of Corporatism on Macroeconomic Performance and Industrial Disputes in 18 Industrialized Democracies, in «Comparative Political Studies», 25, pp. 139-168.

- (1996), Constitutional Structure and Regime Performance in 18 Industrialized Democracies: A Test of Olson's Hypothesis, in «European Journal of Political Research», 29, pp. 87-104.

Crouch, C. (1985), Conditions for Trade Union Wage Restraint, in L.N. Lindberg e C.S. Maier (a cura di), The Politics of Inflation and Economic Stagnation, Washington, Brookings Institution, pp. 105-139.

Dahrendorf, R. (1989), Tertium non-Datur: A Comment on the Andrew Shonfield Lectures, in «Government and Opposition», 24, pp. 131-141.

Downs, A. (1957), An Economic Theory of Democracy, New York, Harper and Row.

Edin, P.A. e H. Ohlsson (1991), Political Determinants of Budget Deficits: Coalition Effects versus Minority Effects, in «European Economic Review», 35, pp. 1597-1603.

EJPR (vari anni), Political Data Yearbook, Dordrecht, Kluwer Academic Publishers.

ES (vari anni), National Elections, in «Electoral Studies».

Ferner, A. e R. Hyman (a cura di) (1992), Industrial Relations in the New Europe, London, Blackwell.

Finer, S.E. (1975), Introduction: Adversary Politics and Electoral Reform, in S.E. Finer (a cura di), Adversary Politics and Electoral Reform, London, Anthony Wigram.

Gallagher, M., M. Laver e P. Mair $\left(1995^{2}\right)$, Representative Government in Modern Europe, New York, McGraw-Hill.

Garrett, G. e P. Lange (1989), Government Partisanship and Economic Performance: When and How Does «Who Governs» Matter?, in «Journal of Politics», 51, pp. 676-693.

Goldberger, A.S. (1968), Topics in Regression Analysis, New York, Macmillan.

Goldthorpe, J.H. (a cura di) (1984), Order and Conflict in Contemporary Capitalism, Oxford, Oxford University Press.

Gorvin, I. (a cura di) (1989), Elections since 1945: A Worldwide Reference Compendium, Chicago, St. James.

Gualmini, E. (1997), L'evoluzione degli assetti concertativi in Italia e in 
Germania, in «Rivista Italiana di Scienza Politica», 27, pp. 101150.

Hibbs, D.A. Jr. (1977), Political Parties and Macroeconomic Policy, in «American Political Science Review», 71, pp. 1467-1487.

Hicks, A. (1988), Social Democratic Corporatism and Economic Growth, in «Journal of Politics», 50, pp. 677-704.

Hicks, A. e J. Misra (1993), Political Resources and the Growth of Welfare in Affluent Capitalist Democracies, in «American Journal of Sociology», 99, pp. 668-710.

Hicks, A. e D.W. Patterson (1989), On the Robustness of the Left Corporatist Model of Economic Growth, in «Journal of Politics», 51, pp. 662-675.

Hicks, A., D.H. Swank e M. Ambuhl (1989), Welfare Expansion Revisited: Policy Routines and their Mediation by Parties, Class and Crisis, 1957-1982, in «European Journal of Political Research», 17, pp. 401-430.

Hollingsworth, J.R. e W. Streeck (1994), Countries and Sectors. Concluding Remarks on Performance, Convergence, and Competitiveness, in J.R. Hollingsworth, P.C. Schmitter e W. Streeck (a cura di), Governing Capitalist Economies. Performance and Control of Economic Sectors, Oxford, Oxford University Press, pp. 270-301.

Huber, E., C. Ragin e J.D. Stephens (1993), Social Democracy, Christian Democracy, Constitutional Structure, and the Welfare State, in «American Journal of Sociology», 99, pp. 711-749.

Immergut, E. (1992), The Political Construction of Interests: National Health Insurance Politics in Switzerland, France and Sweden, 19301970, London, Cambridge University Press.

Jackman, R.W. (1989), The Politics of Economic Growth, Once Again, in «Journal of Politics», 51, pp. 646-661.

Jankowski, R. (1988), Preference Aggregation in Political Parties and Interest Groups: A Synthesis of Corporatist and Encompassing Organization Theory, in «American Journal of Political Science», 32, pp. 105-125.

Katz, R.S. e P. Mair (a cura di) (1992), Party Organizations: A Data Handbook, London, Sage.

- (1994), How Parties Organize. Change and Adaptation in Party Organizations in Western Democracies, London, Sage.

Keech, W.R., R.H. Bates e P. Lange (1991), Political Economy within Nations, in W. Crotty (a cura di), Political Science: Looking to the Future, vol. 2, Evanston, Northwestern University Press, pp. 219. 263.

Keesings Publications (vari anni), Keesings Contemporary Archives, London, Longman.

Keman, H. (1993), The Politics of Managing the Mixed Economy, in H. Keman (a cura di), Comparative Politics: New Directions in Theory and Method, Amsterdam, VU Press, pp. 161-189. 
Keman, H., H. Paloheimo e P. Whiteley (a cura di) (1987), Coping with the Economic Crisis, London, Sage.

Korpi, W. (1983), The Democratic Class Struggle, London, Routledge $\&$ Kegan Paul.

Korpi, W. e M. Shalev (1979), Strikes, Industrial Relations and Class Conflict in Capitalist Societies, in «British Journal of Sociology», 30, pp. 127-171.

Kriesi, H. (1994), Les democraties Occidentales, Paris, Economica.

Laakso, M. e R. Taagepera (1979), Effective Number of Parties: A Measure with Application to Western Europe, in «Comparative Political Studies», 12, pp. 3-27.

Lange, P. e H. Meadwell (1991), Typologies of Democratic Systems: From Political Inputs to Political Economy, in H.J. Wiarda (a cura di), New Directions in Comparative Politics, Boulder, Westview Press, pp. 80-112.

Lehmbruch, G. (1985), Neo-Corporatism in Western Europe: A Reassessment of the Concept in Cross-National Perspective, relazione al $13^{\circ}$ Congresso mondiale dell' IPSA, 15-19 giugno, Paris, pp. 1-27.

Lehmbruch, G. e P.C. Schmitter (a cura di) (1982), Patterns of Corporatist Policy-Making, London, Sage.

Lehner, F. (1988), The Political Economy of Distributive Conflict, in Castles, Lehner e Schmidt, pp. 54-96.

Lijphart, A. (1975), The Comparable-Cases Strategy in Comparative Research, in «Comparative political Studies», 8, pp. 158-177.

- (1977), Democracy in Plural Societies: A Comparative Exploration, New Haven, Yale University Press.

- (1984), Democracies. Patterns of Majoritarian and Consensus Government in Twenty-One Countries, New Haven, Yale University Press; trad. it. Le democrazie contemporanee, Bologna, Il Mulino, 1988.

- (1991), Constitutional Choices for New Democracies, in «Journal of Democracy», 2, pp. 72-84.

- (1994a), Democracies: Forms, Performance, and Constitutional Engineering, in «European Journal of Political Research», 25, pp. 1-17.

- (1994b), Electoral Systems and Party Systems. A Study of TwentySeven Democracies, 1945-1990, Oxford, Oxford University Press.

Lijphart, A. e M.M.L. Crepaz (1991), Corporatism and Consensus Democracy in Eighteen Countries: Conceptual and Empirical Linkages, in «British Journal of Political Science», 21, pp. 235-246.

Magagna, V. (1988), Representing Efficiency: Corporatism and Democratic Theory, in «Review of Politics», 50, pp. 420-444.

Morlino, L. (1998), Democracy between Consolidation and Crisis: Parties, Groups and Citizenship in Southern Europe, Oxford, Oxford University Press.

OECD (1997), Oecd Economic Outlook, n. 61, Paris, Oecd.

- (vari anni), Oecd Economic Outlook, Paris, Oecd. 
Olson, M. (1965), The Logic of Collective Action, Cambridge, Harvard University Press.

- (1982), The Rise and Decline of Nations: Economic Growth, Stagflation, and Social Rigidities, New Haven, Yale University Press, trad. it. Ascesa e declino delle nazioni, Bologna, Il Mulino.

- (1986), A Theory of the Incentives Facing Political Organizations: Neo-Corporatism and the Hegemonic State, in «International Political Science Review», 7, pp. 165-189.

Papadopoulos, Y. (1995), Democrazia e referendum, in «Rivista Italiana di Scienza Politica», 25, pp. 197-226.

Pappalardo, A. (1991), Austerità conservatrice e rigore socialista: Che differenza? I, in «Rivista Italiana di Scienza Politica», 21, pp. 33-90.

Pappalardo, A. e L. Mattina (1998), Democrazie e decisioni, Roma, NIS.

Peele, G. (1988), British Conservatism: Ideological Change and Electoral Uncertainty, in B. Girvin (a cura di), The Transformation of Contemporary Conservatism, London, Sage, pp. 13-35.

Pekkarinen, J. (1992), Corporatism and Economic Performance in Sweden, Norway, and Finland, in Pekkarinen, Pohjola, Rowthorn, pp. 298-337.

Pekkarinen, J., M. Pohjola e B. Rowthorn (a cura di) (1992), Social Corporatism: A Superior Economic System?, Oxford, Clarendon Press.

Pierson, P. (1994), Dismantling the Welfare State? Reagan, Thatcher and the Politics of Retrenchment, London, Cambridge University Press.

- (1996), La nuova politica del welfare state: un'analisi comparata degli interventi restrittivi, in «Stato e Mercato», 46, pp. 3-50.

Robertson, D. (1984), Adversary Politics, Public Opinion and Electoral Cleavages, in D. Kavanagh e G. Peele (a cura di), Comparative Government and Politics. Essays in Honour of S. E. Finer, Boulder, Westview Press, pp. 214-241.

Roubini, N. e J. Sachs (1989a), Political and Economic Determinants of Budget Deficits in the Industrial Democracies, in «European Economic Review», 33, pp. 903-938.

- (1989b), Government Spending and Budget Deficits in the Industrial Countries, in «Economic Policy», 8, pp. 100-132.

Rowthorn, R. e A. Glyn (1990), The Diversity of Unemployment Experiences since 1973, in S.A. Marglin e G.B. Schor (a cura di), The Golden Age of Capitalism, Oxford, Oxford University Press, pp. 19-47.

Sartori, G. (1974), Tecniche decisionali e sistema dei comitati, in «Rivista Italiana di Scienza Politica», 4, pp. 3-45.

- (1976), Parties and Party Systems. A Framework for Analysis, London, Cambridge University Press.

- (1982), Rivisitando il pluralismo polarizzato, in G. Sartori, Teoria dei partiti e caso italiano, Milano, Sugarco, pp. 193-214. 
- (1984), Le «leggi» sull'influenza dei sistemi elettorali, in «Rivista Italiana di Scienza Politica», 14, pp. 3-40.

Scharpf, F.W. (1991), Political Institutions, Decision Styles, and Policy Choices, in R. Czada e A. Windhoff-Héritier (a cura di), Political Choice, Institutions, Rules and the Limits of Rationality, Frankfurt am Mainz/Boulder, Campus/Westview, pp. 53-86.

Schick, A. (1993), Governments versus Budget Deficits, in Weaver e Rockman, pp. 187-236.

Schmidt, M.G. (1988), The Politics of Labour Market Policy, in Castles, Lehner e Schmidt, pp. 4-53.

- (1996), When Parties Matter: A Review of the Possibilities and Limits of Partisan Influence on Public Policy, in «European Journal of Political Research», 30, pp. 155-183.

Schmitter, P.C. (1974), Still the Century of Corporatism?, in «Review of Politics», 36, pp. 85-131.

- (1981), Interest Intermediation and Regime Governability in Contemporary Europe and North America, in Berger, pp. 287-330.

- (1989), Corporatism is Dead! Long Live Corporatism, in «Government and Opposition», 24, pp. 54-73.

Schüttemeyer S.S., (1994), Hierarchy and Efficiency in the Bundestag: The German Answer for Institutionalizing Parliament, in G.W. Copeland e S.C. Patterson (a cura di), Parliaments in the Modern World, Ann Arbor, University of Michigan Press, pp. 29-58.

Siniscalco, D. e G. Tabellini (1993), Efficienza e rappresentanza del sistema elettorale, in «il Mulino», n. 4, pp. 810-819.

Steinmo, S. (1989), Political Institutions and Tax Policy in the United States, Sweden, and Britain, in «World Politics», 61, pp. 500-535.

Streeck, W. (1984), Neo-Corporatist Industrial Relations and the Economic Crisis in West Germany, in Goldthorpe, pp. 291-314.

Streeck, W. (1994), Pay Restraint without Incomes Policy: Institutionalized Monetarism and Industrial Unionism in Germany, in R. Dore, R. Boyer e Z. Mars (a cura di), The Return to Incomes Poli$c y$, London, Pinter, pp. 118-140.

Streeck, W. e P.C. Schmitter (a cura di) (1985), Private Interest Government. Beyond Market and State, London, Sage.

Strom, K. (1990), Minority Government and Majority Rule, New York, Cambridge University Press.

Swank, D.H. (1988), The Political Economy of Government Domestic Expenditures in the Affluent Democracies, 1960-1980, in "American Journal of Political Science», 32, pp. 1120-1150.

Therborn, G. (1992), Lessons from 'Corporatist' Theorizations, in Pekkarinen, Pohjola e Rowthorn, pp. 24:43.

Tufte, E.R. (1978), Political Control of the Economy, Princeton, Princeton University Press.

Van Deth, J.W. (1995), Comparative Politics and the Decline of the Nation-State in Western Europe, in «European Journal of Political Research», 27, pp. 443-462. 
Weaver, R.K. e B.A. Rockman (1993a), When and How Do Institutions Matter?, in Weaver e Rockman, pp. 445-461.

Weaver, R.K. e B.A. Rockman (a cura di) (1993b), Do Institutions Matter? Government Capabilities in the US and Abroad, Washington, Brookings Institution.

Weir, M. e T. Skocpol (1983), State Structure and Social Keynesian Responses to the Great Depression in Sweden and the United States, in «International Journal of Comparative Sociology», 24, pp. 4-29.

Western, B. (1994), Unionization and Labor Market Institutions in Advanced Capitalism, 1950-1985, in «American Journal of Sociology», 100, pp. 1329-1361.

- (1995), A Comparative Study of Working-Class Disorganization: Union Decline in Eighteen Advanced Capitalist Democracies, in «American Sociological Review», 60, pp. 165-191.

Woldendorp, J., H. Keman e I. Budge (1993) Political Data 19451990, in «European Journal of Political Research», 24, pp. 1-120. 